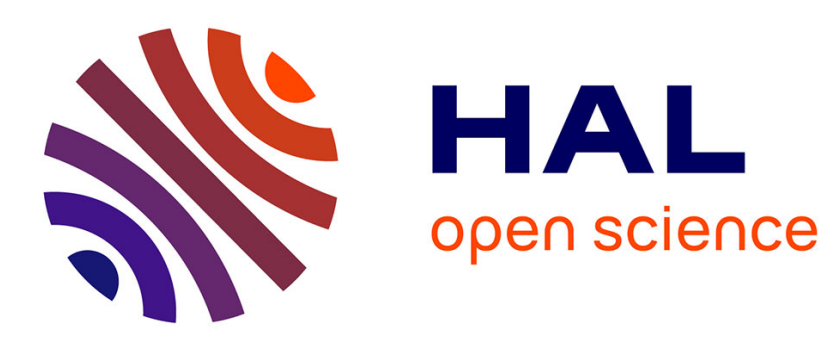

\title{
Large eddy simulation of turbulent buoyant flow in a confined cavity with conjugate heat transfer
}

\author{
C. Cintolesi, A. Petronio, V. Armenio
}

\section{To cite this version:}

C. Cintolesi, A. Petronio, V. Armenio. Large eddy simulation of turbulent buoyant flow in a confined cavity with conjugate heat transfer. Physics of Fluids, 2015, 27 (9), pp.095109. 10.1063/1.4930815 . hal-02070280

\section{HAL Id: hal-02070280 \\ https://hal.science/hal-02070280}

Submitted on 17 Mar 2019

HAL is a multi-disciplinary open access archive for the deposit and dissemination of scientific research documents, whether they are published or not. The documents may come from teaching and research institutions in France or abroad, or from public or private research centers.
L'archive ouverte pluridisciplinaire HAL, est destinée au dépôt et à la diffusion de documents scientifiques de niveau recherche, publiés ou non, émanant des établissements d'enseignement et de recherche français ou étrangers, des laboratoires publics ou privés. 


\section{AIP $\mid \begin{aligned} & \text { Physiso of } \\ & \text { Fluids }\end{aligned}$}

\section{Large eddy simulation of turbulent buoyant flow in a confined cavity with conjugate heat transfer}

C. Cintolesi, A. Petronio, and V. Armenio

Citation: Physics of Fluids 27, 095109 (2015); doi: 10.1063/1.4930815

View online: http://dx.doi.org/10.1063/1.4930815

View Table of Contents: http://scitation.aip.org/content/aip/journal/pof2/27/9?ver=pdfcov

Published by the AIP Publishing

\section{Articles you may be interested in}

A priori and a posteriori investigations for developing large eddy simulations of multi-species turbulent mixing under high-pressure conditions

Phys. Fluids 27, 035117 (2015); 10.1063/1.4916284

Direct numerical simulation of turbulent heat transfer in a fluid-porous domain

Phys. Fluids 25, 125110 (2013); 10.1063/1.4851416

Numerical studies of the effects of large neutrally buoyant particles on the flow instability and transition to turbulence in pipe flow

Phys. Fluids 25, 043305 (2013); 10.1063/1.4802040

Heat transfer in turbulent channel flow over protrusions

AIP Conf. Proc. 1479, 2032 (2012); 10.1063/1.4756588

Large-eddy simulations of turbulent heat transfer in stationary and rotating square ducts

Phys. Fluids 14, 2804 (2002); 10.1063/1.1489684

\section{Did your publisher get}

18 MILLION DOWNLOADS in 2014?

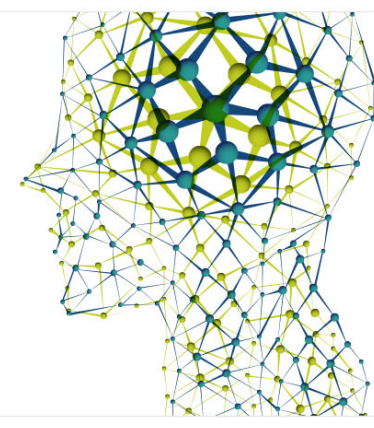




\title{
Large eddy simulation of turbulent buoyant flow in a confined cavity with conjugate heat transfer
}

\author{
C. Cintolesi, ${ }^{1, a)}$ A. Petronio, ${ }^{2, b)}$ and V. Armenio ${ }^{1, c)}$ \\ ${ }^{1}$ DIA-University of Trieste, Piazzale Europa 1, I-34127 Trieste, Italy \\ ${ }^{2}$ IEFLUIDS s.r.l., Piazzale Europa 1, I-34127 Trieste, Italy
}

(Received 4 June 2015; accepted 31 August 2015; published online 18 September 2015)

\begin{abstract}
Turbulent natural convection in enclosure is a paradigmatic case for wide class of processes of great interest for industrial and environmental problems. The solid-fluid thermal interaction, the anisotropy of the turbulence intensity in the flow field along with the transient nature of heat transfer processes, pose challenges regarding the numerical modeling. The case of a square cavity with differently heated vertical walls and two horizontal conductive plates is studied at $R a=1.58 \times 10^{9}$. The study is carried out numerically, using large-eddy simulation together with a dynamic Lagrangian turbulence model and a conjugate heat transfer method to take into account heat transfer at the solid surfaces. First, validation is carried out against the literature experimental and numerical data. The results of validation tests evidence the limitations of using the adiabatic conditions as a model for reproducing an insulator. In fact, the adiabatic condition represents the asymptotic behavior which is often difficult to reach in real conditions. Successively, the model is used to investigate the effect on the flow field of different materials composing the horizontal walls. Initial conditions representative of physical experiment are used. In order to reduce the computational time required for a simulation with insulating materials at the walls, a four-step temperature advancement strategy is proposed, based on the artificial reduction-first and recover-later of the specific heat coefficient $C_{p}$ of the materials at different stages of the simulation. The conductivity of the solid media is found to influence the flow configuration since heat transfer at the solid walls substantially modifies the turbulent field and makes the flow field less homogeneous along the horizontal direction. (C) 2015 AIP Publishing LLC. [http://dx.doi.org/10.1063/1.4930815]
\end{abstract}

\section{INTRODUCTION}

Turbulent natural convection in closed volumes is one of the most important subjects in applied physics both from a theoretical perspective and for implications in engineering systems.

In these systems, a temperature gradient, for example, originated by differently heated walls, gives rise to buoyancy forces driving the fluid and triggering turbulence.

The correct modeling and reproduction of such flows is of great interest both in environmental flows, like convective boundary layer or the convective motion ruled by heating of vertical walls of skyscrapers at neighbor scale, as in industrial applications. In the latter cases, among the others, natural convection rules (with increasing Rayleigh and Reynolds numbers) the process of cooling of electrical equipments or collectors of solar energy, the efficiency in building ventilation, and the energy efficiency in home appliances.

Neglecting the solid-fluid thermal coupling in the above mentioned applications may result in an oversimplification of the system itself, eventually leading to incorrect results. Moreover, the transient nature of the heat transfer process along with the complexity of the geometry and the

\footnotetext{
a)Electronic mail: carlo.cintolesi@gmail.it

b) Electronic mail: a.petronio@iefluids.com

c) Electronic mail: armenio@dica.units.it
} 
anisotropy of turbulent fluctuations poses challenges regarding mathematical modeling. Specifically, coupling between thermal conductive solid walls and convective motion may play a crucial role in the development of the fluid dynamics and the overall process of heat transfer.

The present study aims at assessing the influence of the conductive boundary on the buoyancydriven flow, with a specific focus onto heat coupling mechanism between fluid and solid media.

We consider the differentially heated square cavity (DHSC). It is one of the most investigated, both numerically and experimentally, benchmark cases for the understanding of natural convection. In this system, the flow is, essentially, two-dimensional; it is driven by buoyancy arising from fluid heated by a hot vertical wall and cooled by the opposite one. The vertical walls are considered at constant temperature whereas the horizontal plates are usually assumed adiabatic. Despite the simplicity of geometry and boundary conditions, the system displays all the main features of natural convection in a confined environment and it thus appears as an appropriate test case for the investigation of the solid-fluid media thermal interaction. The high sensitivity of the flow to the boundary conditions requires accurate experimental studies on one side, and accurate mathematical modeling and numerical methods on the other.

Mergui and Penot $^{3}$ analyzed experimentally the DHSC, providing reference data useful to calibrate and validate numerical models. Salat et al. ${ }^{4}$ questioned about the achievement of adiabatic conditions in experiments reported in Ref. 3. They highlighted the strong influence of temperature boundary conditions imposed in numerical models, especially near the horizontal boundaries and in the corner region. In order to match the experimental data of Ref. 3, it was necessary to switch from adiabatic to Dirichlet boundary condition, imposing the temperature experimental profile along the horizontal walls.

Further experiments on the same configuration cavity were carried out by Tian and Karayiannis $^{5,6}$ and Ampofo and Karayiannis, ${ }^{7}$ taking into consideration the conductivity of the horizontal walls. Detailed data about mean quantity profiles and turbulent statistics were provided and are here considered as reference data for the present study.

From a mathematical point of view, the three main mathematical frameworks to study DHSC are, respectively, the Reynolds-Averaged Navier-Stokes Equations (RANSE), Direct Numerical Simulation (DNS), and Large-Eddy Simulation (LES). RANSE has been usually applied considering two-dimensional motion, which is a reasonable assumption when the horizontal aspect-ratio of the cavity is larger than unity. Salat et al. ${ }^{4}$ compared the results of the first two approaches and observed that two-dimensional RANSE simulations are less accurate in reproducing the corner flow and the core stratification, while they give a good description of the general flow field at a relative low computational cost. Other authors indicate additional elements that could be crucial for an accurate simulation: Omri and Galanis ${ }^{8}$ performed RANSE simulations with the shear-stress-transitional $k-\omega$ turbulence model, underlining the importance of using a realistic temperature distribution on the boundary walls; Hsieh and Lien ${ }^{9}$ used an unsteady RANSE approach in conjunction with the $k-\epsilon$ model at low-Re number, emphasizing the computational difficulties of reproducing convective flow with low turbulence intensity and underlining the need of a reliable turbulence model; Ibrahim et al.$^{10}$ recently studied the effect of thermal radiation using a two-dimensional LES (which has to be consider as an unsteady RANSE) and suggested that this phenomenon is responsible for the increased temperature in the core region of the cavity observed in the experimental studies.

Among the more recent numerical studies, we refer to the work of Peng and Davidson ${ }^{11}$ who performed LES with dynamic subgrid-scale (SGS) turbulence model, imposing the experimental temperature profile as boundary condition at horizontal walls. The results were in good agreement with the experimental data although the root-mean square (RMS) statistics were in general overestimated. Bosshard et al. ${ }^{12}$ employed a dynamic model in a LES, carried out by means of a spectral numerical method, together with adiabatic conditions on horizontal boundaries; Zimmermann and Groll ${ }^{13}$ imposed a linear distribution of temperature and considered a compressible approach instead of using the Boussinesq approximation.

In many situations, the exchange of thermal energy between the fluid and the solid media occurs even if strong insulating materials are considered. Hence, the adiabatic condition has to be considered as an idealized condition. To the knowledge of the authors, the thermal coupling between the two media has never been considered to assess the impact of the solid thermal properties 
on the flow developing within the DHSC. From a modeling point of view, this implies the coupled solution of the flow field and the thermal field within the solid, with appropriate conditions at the fluid-solid interface. This problem is referred in literature as Conjugate Heat Transfer (CHT).

A review of the techniques to treat this subject is in the work of Dorfman and Renner, ${ }^{14}$ while Duchaine et al. ${ }^{15,16}$ describe in detail some coupling strategies and analyze their stability properties and efficiency. The CHT was also studied by Tiselj et al. ${ }^{17}$ who carried out a DNS for two-dimensional turbulent channel flow and studied the propagation of the temperature fluctuations inside the wall when different oscillatory boundary conditions are imposed. Garai et al. ${ }^{18}$ extended this latter work, analyzing the convective instability on heat transport in channel flow and the variation into the solid-fluid interface when the physical properties of the solid boundary change.

Although DHSC is a geometrically simple and idealized configuration, it poses some challenging issues:

- the influence of the conductive solid boundaries on the fluid domain cannot be neglected;

- the characteristic diffusion time of temperature within the solid matter can be of some orders of magnitude higher than that in the fluid, meaning that long simulation time is required to reach a statistical steady state;

- accurate turbulence modeling techniques should be used, able to reproduce localized and anisotropic turbulent flow.

Previous researches focused only on one of the above mentioned issues. In the present work, we try considering all issues, applying a reliable and accurate turbulence model together with a thermal coupling technique, allowing for a more realistic study of the flow field as well as the thermal state of the solid conductive boundaries.

The development of the numerical model is carried out within the OpenFOAM ${ }^{\circledR}$ framework, taking advantage of its flexibility and capability to treat complex cases. The large-eddy simulation methodology, together with a Lagrangian dynamic model for sub-grid scale momentum and temperature fluxes, leads to the possibility to investigate accurately the complex inhomogeneous turbulent field developing within the DHSC. The CHT technique allows to represent realistically the fluid-solid coupling across the interface, taking into account for the thermal properties of the solid medium.

The paper is organized as follows: Sec. II presents the mathematical model and the numerical implementation used for simulations; Sec. III describes some non-dimensional parameters suitable for studying this physical problem; Sec. IV describes the DHSC and supplies details on the simulation settings; in Sec. V, the numerical solver for fluid flow is validated against experimental and numerical literature results; in Sec. VI, the temperature advancement strategy is discussed and the implementation of CHT is validated against experimental data; Sec. VII is focused on the analysis of the fluid-thermal field obtained using different conductive materials; in Sec. VIII, concluding remarks are given.

\section{SIMULATION METHODOLOGY}

In the following, the subscripts are used to specify the particular use of a general quantity. If $\phi$ is a generic variable, then $\phi_{f}$ is the fluid related variable; $\phi_{s}$ is the solid related variable; $\phi_{w}$ is the variable evaluated on the wall; $\phi_{\mathrm{SGs}}$ is the sub-grid scale quantity. The International System of Units is used to express the value of all quantities.

\section{A. Mathematical model}

The study of thermal interaction between solid and fluid media involves three main actors: the fluid with its inner dynamics, the diffusion of heat in the solid medium, and the heat transfer at the interface. All these phenomena have to be modeled through a set of appropriate equations. 
In our case, the fluid in the cavity is assumed to be incompressible and the buoyancy force, driven by temperature gradient, is taken into account by the Boussinesq approximation,

$$
\begin{aligned}
\frac{\partial u_{i}}{\partial x_{i}} & =0 \\
\frac{\partial u_{i}}{\partial t}+u_{j} \frac{\partial u_{i}}{\partial x_{j}} & =-\frac{1}{\rho_{0}} \frac{\partial p}{\partial x_{i}}+v \frac{\partial^{2} u_{i}}{\partial x_{j} \partial x_{j}}-\frac{\rho}{\rho_{0}} g \delta_{i 2}, \\
\frac{\rho}{\rho_{0}} & =1-\beta_{T}\left(T-T_{0}\right),
\end{aligned}
$$

where $u$ is the fluid velocity, $p$ is the pressure, $T$ is temperature and $T_{0}$ is the reference one, $\rho$ is the space-time variable density in the fluid flow while $\rho_{0}$ is the reference density, $v$ is the molecular kinematic viscosity, $g$ is the gravity acceleration, and $\beta_{T}$ is the thermal expansion coefficient.

The temperature equation for fluid domain reads as

$$
\frac{\partial T_{f}}{\partial t}+u_{j} \frac{\partial T_{f}}{\partial x_{j}}=\alpha_{f} \frac{\partial^{2} T_{f}}{\partial x_{j} \partial x_{j}},
$$

where we denote with $\alpha$ the molecular thermal diffusivity.

In the solid medium, the space-time temperature evolution has to be modeled. The governing equation is the classical law of diffusion,

$$
\frac{\partial T_{s}}{\partial t}=\alpha_{s} \frac{\partial^{2} T_{s}}{\partial x_{j} \partial x_{j}}
$$

A thermal coupling boundary condition at the solid-fluid interface $\Gamma$ has to be introduced, enforcing the continuity of temperature and the balance of the heat fluxes,

$$
\begin{aligned}
\left.T_{s}\right|_{\Gamma} & =\left.T_{f}\right|_{\Gamma}, \\
k_{s}\left(\frac{\partial T_{s}}{\partial n}\right) & =k_{f}\left(\frac{\partial T_{f}}{\partial n}\right),
\end{aligned}
$$

where $n$ denotes the normal to the surface and $k$ is the thermal conductivity. Different techniques are available in the literature to perform the coupling of the thermal field at the interface (see, among the others Quarteroni and Valli $\left.{ }^{19}\right)$. A Neumann-Neumann decomposition method is here applied, assuring the balance of both (6) and (7) below a prescribed tolerance. More details on the coupling methodology herein employed can be found in the work of Sosnowski et al. ${ }^{20}$

\section{B. Numerical methods}

For the fluid part, a LES approach is used. The computational grid acts on the equations as an implicit spatial filter. In filtered Navier-Stokes equations (2), SGS stress tensor, $\tau_{i j}={\overline{u_{i} u_{j}}}_{\bar{u}} \bar{u}_{i} \bar{u}_{j}$, appears. The straight over-bar denotes the filter width, which in this case is the local cell width computed as $\bar{\Delta}=\left(\bar{\Delta}_{x} \bar{\Delta}_{y} \bar{\Delta}_{z}\right)^{1 / 3}$.

Under the eddy viscosity assumption, it can be modeled as

$$
\tau_{i j}-\frac{\delta_{i j}}{3} \tau_{k k}=-2 v_{\mathrm{sGS}} \overline{S_{i j}},
$$

where $\overline{S_{i j}}=(1 / 2)\left[\left(\partial \bar{u}_{i} / \partial x_{j}\right)+\left(\partial \bar{u}_{j} / \partial x_{i}\right)\right]$ is the strain rate tensor and $v_{\mathrm{SGS}}$ is the sub-grid scale viscosity. Similarly, the filtering of the temperature equation in fluid part (4) gives rise to a SGS flux $h_{i}=\overline{T_{f} u_{i}}-\overline{T_{f}} \overline{u_{i}}$ that can be computed assuming the gradient diffusivity hypothesis,

$$
h_{i}=-\alpha_{\mathrm{sGs}} \frac{\partial \overline{T_{f}}}{\partial x_{i}},
$$

where $\alpha_{\mathrm{SGS}}$ is the sub-grid scale thermal diffusivity. 
The SGS viscosity and diffusivity have to be modeled. Smagorinsky ${ }^{21}$ proposed the following relation to link the eddy viscosity to the strain rate tensor:

$$
v_{\mathrm{SGS}}=c_{s}^{2} \bar{\Delta}^{2}\left|\overline{S_{i j}}\right|,
$$

where $c_{s}^{2}$ is the Smagorinsky constant for SGS momentum fluxes. A similar formula can be given for the SGS diffusivity,

$$
\alpha_{\mathrm{SGS}}=c_{t}^{2} \bar{\Delta}^{2}\left|\overline{S_{i j}}\right|,
$$

where $c_{t}^{2}$ is the Smagorinsky constant for SGS thermal fluxes.

The Smagorinsky constants can be evaluated from analytical considerations, experiments, or DNS. The main drawback of setting a value for the constants relays in the assumption of flow homogeneity. Close to solid boundaries, where the turbulent length-scales decrease, the Van Driest damping function is usually applied to overcome such limitation.

In a non-homogeneous flow, the best suited approach is to determine $c_{s}^{2}$ and $c_{t}^{2}$ dynamically (see Germano et al. ${ }^{22}$ and Lilly ${ }^{23}$ ). Among the possible choices of dynamic models, the use of the Lagrangian dynamic SGS model of Meneveau et $a l .{ }^{24}$ allows to tackle problems without any homogeneity directions. In dynamic models, the sub-grid scale quantities are estimated from the resolved scales using an additional test filter, denoted by an angle bracket over-bar, of width $\widehat{\Delta}=2 \bar{\Delta}$.

The theoretical background of this procedure is the Germano identity that links the sub-grid scale tensors computed using the two different filters,

$$
L_{i j}=\mathcal{T}_{i j}-\widehat{\tau}_{i j}=\widehat{\bar{u}}_{i} \bar{u}_{j}-\widehat{\bar{u}}_{i} \widehat{\bar{u}}_{j},
$$

then, the stress tensors of the large and small scales can be modeled by the Smagorinsky eddyviscosity model, leading to

$$
\widehat{\tau}_{i j}={\widehat{\overline{u_{i} u}}}_{j}-{\widehat{\overline{u_{i}}}}_{j} \cong-2 c_{s}^{2} \bar{\Delta}^{2} \mid \overline{\bar{S}}_{\bar{S}}
$$

and

$$
\mathcal{T}_{i j}={\widehat{\overline{u_{i}}}}_{j}-\widehat{\overline{\bar{u}}}_{i} \widehat{\bar{u}}_{j} \cong-2 c_{s}^{2}(2 \bar{\Delta})^{2}|\widehat{\bar{S}}| \widehat{\bar{S}}_{i j}
$$

Inserting the latter in (12) gives an over-determined system of equations for the unknown $c_{s}$. In order to solve the system, we should minimize the error associated to the use of Smagorinsky approximation, defined as

$$
e_{i j}=L_{i j}-2 \bar{\Delta}^{2}\left[c_{s}^{2} \mid{\widehat{\bar{S}} \mid \overline{S_{i j}}}_{i}-4 c_{s}^{2} \widehat{\left.\bar{S} \mid \overline{\bar{S}}_{i j}\right]}\right.
$$

The Lagrangian model is obtained by minimizing this error (in a least-square sense) under the assumption that $c_{s}$ does not vary along the fluid-particle Lagrangian trajectories, and the coefficient is determined as

$$
c_{s}^{2}=\frac{\mathcal{I}_{L M}(\mathbf{x}, t)}{\mathcal{I}_{M M}(\mathbf{x}, t)},
$$

where the numerator and denominator can be expressed by integrals arising from the minimization procedure and that are solutions of the following equations:

$$
\begin{gathered}
\frac{\partial \mathcal{I}_{L M}}{\partial t}+\bar{u}_{k} \frac{\partial \mathcal{I}_{L M}}{\partial x_{k}}=\frac{1}{\mathrm{~T}}\left(L_{i j} M_{i j}-\mathcal{I}_{L M}\right), \\
\frac{\partial \mathcal{I}_{M M}}{\partial t}+\bar{u}_{k} \frac{\partial \mathcal{I}_{M M}}{\partial x_{k}}=\frac{1}{\mathrm{~T}}\left(M_{i j} M_{i j}-\mathcal{I}_{M M}\right) .
\end{gathered}
$$

The relaxation time scale is assumed to be

$$
\mathrm{T}=\theta \bar{\Delta}\left(\mathcal{I}_{L M} \mathcal{I}_{M M}\right)^{-1 / 8}, \quad \theta=1.5
$$

and

$$
M_{i j}=2 \bar{\Delta}^{2}\left(\widehat{\overline{|S| \bar{S}_{i j}}}-4|\widehat{\bar{S}}| \overline{\bar{S}}_{i j}\right)
$$


Armenio and Sarkar ${ }^{25}$ showed that the constant $c_{t}^{2}$ can be computed in analogous way: the tensors $L_{i j}$ and $M_{i j}$ are replaced by the similar expressions for heat flux, respectively,

$$
E_{j}=\widehat{\bar{u}_{j} \bar{T}}-\widehat{\bar{u}}_{j} \widehat{\bar{T}}
$$

and

$$
Q_{j}=2 \bar{\Delta}^{2}\left(\widehat{|\bar{S}| \frac{\partial \bar{T}}{\partial x_{j}}}-4|\overline{\bar{S}}| \frac{\partial \hat{\bar{T}}}{\partial x_{j}}\right) .
$$

Then, the factors $\mathcal{J}_{E Q}, \mathcal{J}_{Q Q}$ are computed through equations analogous to (15) and (16), using formula (17) for the calculation of relaxation time. After this, the Smagorinsky constant for thermal fluxes is obtained by

$$
c_{t}^{2}=2 \frac{\mathcal{J}_{E Q}(\mathbf{x}, t)}{\mathcal{J}_{Q Q}(\mathbf{x}, t)} .
$$

The standard Smagorinsky model is employed for comparison purposes, with the settings reported by Peng and Davidson, ${ }^{11}$ namely, $c_{s}^{2}=0.044$. In this case, the Reynolds analogy for the SGS eddy diffusivity of temperature $\alpha_{\mathrm{sGs}}=v_{\mathrm{sGs}} / P r_{t}$, with a turbulent Prandtl number equal to $P r_{t}=0.4$. The Van Driest damping function is used to reduce the turbulent length-scales near the walls.

\section{Algorithm and implementation}

The numerical implementation is carried out taking advantage of the open source $\mathrm{C}++$ library OpenFOAM version 2.1.0.

The code works with unstructured meshes using a finite volume method. The solution of equations is performed using the Pressure-Implicit with Splitting of Operators (PISO), as formulated in most OpenFOAM solvers, based on the work of Issa ${ }^{26}$ and Issa et al. ${ }^{27}$ As discussed, a Lagrangian dynamic model for sub-grid scales, both for momentum and scalar transport, is implemented for the present study while the thermal coupling procedure at the interface is that of the work of Sosnowski et al. ${ }^{20}$

First, the temperature equation is solved for fluid and for solid domains, and an iterative procedure performs the thermal coupling between the two media. Then, the Navier-Stokes equations are solved according to the PISO algorithm. In order to improve numerical stability, the buoyancy term is included in the pressure solution.

The governing equations are discretized in space using a second-order central difference scheme; time integration is performed using an implicit Euler backward differencing scheme which uses the variables at the previous two time steps, resulting in second order accuracy in time (more details are in the OpenFOAM official Programmer's Guide). Overall, the algorithm is second-order accurate both in time and in space.

\section{NON-DIMENSIONAL PARAMETERS}

Parameters characterizing the physics of natural convection are Prandtl number, the ratio between momentum diffusivity, and thermal diffusivity,

$$
\operatorname{Pr}=\frac{v}{\alpha}
$$

Rayleigh number, ratio between buoyancy, and viscous forces,

$$
R a=\frac{g \beta_{T} \Delta T L^{3} P r}{v^{2}}
$$

where $L$ is the system characteristic length. 
For transient phase problems, the characteristic diffusion time $\tau$ of the solid materials is defined. It is a measure of the time required to reach thermal equilibrium,

$$
\tau=\frac{L^{2}}{\alpha}=\frac{\rho C_{p} L^{2}}{k},
$$

where $\alpha=k /\left(\rho C_{p}\right), k$ is the heat conductivity coefficient, and $C_{p}$ is the specific heat coefficient at constant pressure.

Moreover, Garai et al. ${ }^{18}$ use the thermal activity ratio (TAR), a parameter which quantifies heat transfer effects through the interface,

$$
T A R=\sqrt{\frac{\left(k \rho C_{p}\right)_{\text {fluid }}}{\left(k \rho C_{p}\right)_{\text {solid }}}} .
$$

This is the ratio between the thermal effusivity of the fluid and of the solid medium, respectively. The effusivity of a material determines the behavior of the interface in presence of transient heat sources (see the work of Marín ${ }^{28}$ ). From a theoretical point of view, this quantity can be found in the analytical solution of the infinitely fast flame problem, reported also by Duchaine et al. ${ }^{16}$

The equation of heat flux $q_{i f f}$ at the interface between a semi-infinite solid (at temperature $T_{1}$ ) and a semi-infinite gas (at temperature $T_{2}$ ) reads as

$$
q_{\text {iff }}(t)=\frac{T_{2}-T_{1}}{1+T A R} \sqrt{\frac{\left(k \rho C_{p}\right)_{g a s}}{\pi t}},
$$

that is, a rearrangement of equation (4), p. 553 of Ref. 16. From Equation (26), it appears that large values of TAR correspond to weaker heat flux across the interface. Conversely, smaller values of TAR imply a larger heat flux and hence a stronger interaction between solid and fluid.

To better understand the meaning of thermal effusivity and diffusion time, we can consider the solution of the heat equation for a semi-finite homogeneous solid, i.e., a solid bounded by the plane $x=0$ and extending to infinity along the positive $x$ direction. In such a way, the problem is reduced to one dimension, and it is an interesting idealized case of the heat diffusion on the solid boundaries of our cavity. If the initial temperature of the solid is $T_{2}$ and we apply a constant temperature $T_{1}$ over its surface, the analytical solution ${ }^{1}$ for the heat flux $q$ is

$$
q(x, t)=\frac{1}{\sqrt{k \rho C_{p}}} \frac{\left(T_{2}+T_{1}\right)}{\sqrt{\pi t}} e^{-\frac{x^{2}}{2 \alpha t}} .
$$

Equation (27) shows that the heat flux at the surface is inversely proportional to the thermal effusivity of the solid. Moreover, the thermal energy is diffused according to a Gaussian law, both in time and space. The quantity $\ell=2 \sqrt{\alpha t}$ is the diffusion length-scale of the solid: if $\ell \gg L$, the temperature distribution at a distance $L$ from the surface is almost time-independent. Hence, to reach the thermal equilibrium a time,

$$
t \gg \frac{L^{2}}{4 \alpha}=\frac{\tau}{4}
$$

is required.

\section{PROBLEM DESCRIPTION AND SETTINGS}

The general problem of natural convection is studied for the case of square cavity. Specifically, we reproduce the experiment of Tian and Karayiannis ${ }^{5,6}$ and we refer to the simulations of Peng and Davidson ${ }^{11}$ for validation of numerical solver for fluid phase.

\section{A. Case geometry}

The experimental cavity considered in Refs. 5 and 6 has dimension of $0.75 \mathrm{~m} \times 0.75 \mathrm{~m} \times$ $1.5 \mathrm{~m}$. The fluid motion is, on average, two dimensional and develops in the $x, y$-plane, while the 

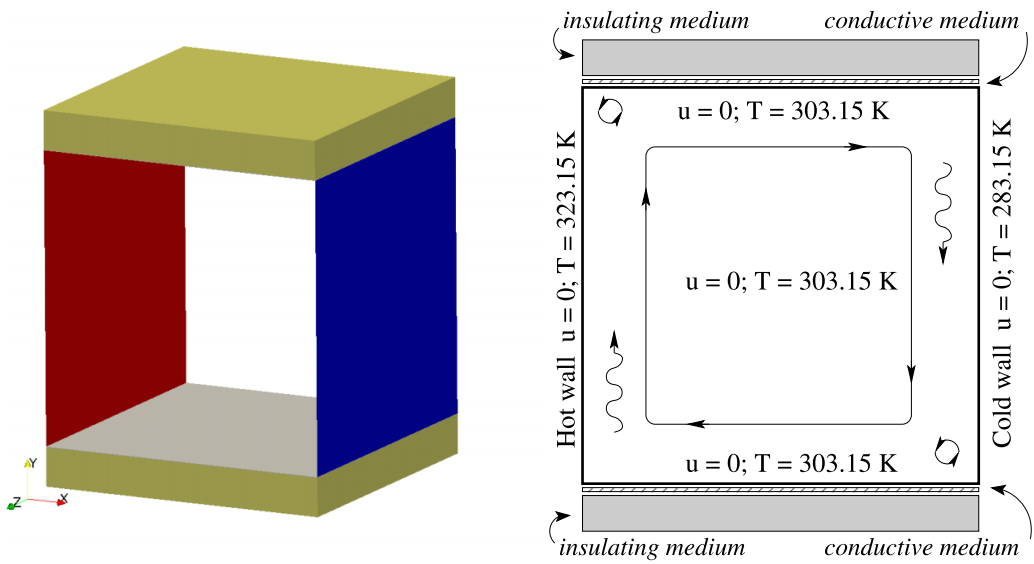

FIG. 1. Problem sketch.

spanwise $z$-direction is of homogeneity for the turbulent flow. The cavity is surrounded by four narrow sheets of conductive boundaries made of mild steel, and two guard cavities on the faces normal to the $z$-direction. The left and right vertical walls have a thickness of $0.006 \mathrm{~m}$ and are maintained at a constant temperature of $T_{h}=323.15 \mathrm{~K}\left(50^{\circ} \mathrm{C}\right)$ and $T_{c}=283.15 \mathrm{~K}\left(10^{\circ} \mathrm{C}\right)$, respectively. The two horizontal walls, with a thickness $H=0.0015 \mathrm{~m}$, are free to conduct heat and are surrounded by a thick insulator. This consist of a polystyrene block of thickness $0.1 \mathrm{~m}$ that is covered with an additional wood plate. Room temperature is maintained at $T=303.15 \mathrm{~K}\left(30^{\circ} \mathrm{C}\right)$, chosen to be equal to the enclosure average temperature. The controlled ambient temperature and the guard cavities are designed to maintain the minimum mass and heat transfer across the cavity. More details of experiment settings can be found in Ref. 5 .

Peng and Davidson ${ }^{11}$ reproduced this experiment numerically, but the heat-transfer process between solids and fluid was not considered. Therefore, the simulations performed by the authors took into account the fluid domain in the cavity only, replacing the solid envelop with suitable boundary conditions. In the spanwise direction, a periodic condition is used. The computational domain was reduced to form a cubic domain of edge $L=0.75 \mathrm{~m}$, after proving that correlation among quantities decay to negligible values after just half domain size in the homogeneous direction.

The computational domain is sketched in Fig. 1. In a preliminary simulation, all the solid elements were taken into account, except for the external wood plates for which details were not provided in the reference article. Then, the two vertical sheets of mild steel were not considered and, thus, replaced by suitable boundaries conditions for the fluid domain. In fact, it turned out that the interposition of the solid bodies between the isothermal source of temperature and the fluid, did not produce any observable effects either on the flow field or within the solid medium. This is due to the fact that the solid boundaries are made of a very narrow slice of high conducting material and the temperature difference from the isotherm boundary and the interior of the cavity is large. For this reason, we choose to avoid the thermal-coupling between the fluid and the vertical sheets, replacing it with a constant temperature condition on the boundaries of the fluid domain.

In order to perform faster simulations, and following, ${ }^{11}$ the domain size in the spanwise direction was $L_{z}=0.75 \mathrm{~m}$, thus reduced by a factor 2 with respect to the reference experiment. This was estimated to be long enough to capture the flow features using periodic boundary conditions along the homogeneous direction.

The origin of axes is located in the bottom left corner of the square and $x, y$ are the horizontal and vertical directions, respectively.

\section{B. Computational domain}

The mesh of the fluid domain is generated as in the work of Peng and Davidson. ${ }^{11}$ The internal part of the cavity is discretized using $96 \times 96 \times 64$ grid cells, regular along the spanwise 
direction and stretched near the walls in the $x, y$-plane. A double-side stretching function, based on hyperbolic tangent, is used,

$$
r(\xi)=\frac{1}{2}\left(1+\frac{\tanh (\delta(\xi-1 / 2))}{\tanh (\delta / 2)}\right),
$$

with a stretching factor $\delta=6.5$ in such way to have 12 computational nodes within $r^{+}<10$, where $r$ is the wall-normal coordinate.

The two horizontal conductive bodies are considered and discretized with a grid of $96 \times 5 \times 64$ cells. The grids are stretched along the vertical direction using Equation (28). Stretching is performed in such a way that the adjacent cells of the interface on the solid and fluid parts have the same size.

The insulator blocks are discretized by $96 \times 30 \times 64$ grid cells. As for the conductors, the mesh is stretched in order to match the cells on the interfaces between two different domains.

\section{Characteristic parameters and physical coefficients}

The fluid considered is air at $303.15 \mathrm{~K}\left(30^{\circ} \mathrm{C}\right)$; Table I lists the physical parameters used. The Rayleigh number is $R a=1.58 \times 10^{9}$, while the parameters for the conductive solid medium are those of the mild steel reported in Table II in Sec. VII.

The coordinates are made non-dimensional with the characteristic length $L=0.75 \mathrm{~m}$. Other characteristic scales of the problem are the buoyant velocity $U_{0}=\sqrt{g \beta_{T} \Delta T L} \cong 1 \mathrm{~m} / \mathrm{s}$, the characteristic time $t_{0}=L / \sqrt{g \beta_{T} \Delta T L} \cong 0.75 \mathrm{~s}$, and the difference of temperature between the two vertical isothermal walls $\Delta T=\left(T_{h}-T_{c}\right)=40 \mathrm{~K}$.

The statistics presented are performed both in space (along the homogeneous direction) and time, when not otherwise specified. Given a generic variable $\phi$, the notation $\langle\phi\rangle$ always represents the average along the homogeneous $z$-direction and in time. This latter, for a time interval equal to about three turnover times of the main vortex in the cavity, is roughly estimated as $t_{\text {ave }} \cong 60 t_{0}$. To calculate the root-mean square resolved quantities, the resolved fluctuations are computed $\phi^{\prime}=\phi-\langle\phi\rangle$, then the root-mean square is $[\phi]_{r m s}=\sqrt{\left\langle\phi^{\prime 2}\right\rangle}$.

\section{Validation: initial and boundary conditions}

The validation of the numerical model is carried out against the simulation data of Peng and Davidson ${ }^{11}$ as well as the experimental data of Ampofo and Karayiannis ${ }^{7}$ and of Tian and Karayiannis. ${ }^{5}$

Two classes of simulations are performed: first, the fluid flow solver is validated in absence of conjugate heat transfer at the interfaces, then, thermal coupling is considered and the temperature distribution on solid media is compared with experimental data.

For the validation of fluid solver, a non-slip condition is prescribed at the four walls, while the initial internal flow starts from rest. Solid media are replaced by suitable boundary conditions: constant temperatures $T_{h}$ and $T_{c}$ are set, respectively, for the left and right vertical boundaries of the cavity. On the horizontal boundaries, the temperature distribution provided by the

TABLE I. Physical parameters of air at $303.15 \mathrm{~K}\left(30^{\circ} \mathrm{C}\right)$.

\begin{tabular}{lll}
\hline \hline$v$ & $\mathrm{~m}^{2} / \mathrm{s}$ & \\
$\alpha$ & $\mathrm{m}^{2} / \mathrm{s}$ & $1.575 \times 10^{-5}$ \\
$\rho$ & $\mathrm{kg} / \mathrm{m}^{3}$ & $2.219 \times 10^{-5}$ \\
$C_{p}$ & $\mathrm{~J} /(\mathrm{kg} \mathrm{K})$ & 1.165 \\
$k$ & $\mathrm{~W} /(\mathrm{m} \mathrm{K})$ & $1.005 \times 10^{3}$ \\
$\beta_{T}$ & $1 / \mathrm{K}$ & 0.026 \\
$P r_{t}$ & $\cdots$ & $3.33 \times 10^{-3}$ \\
\hline \hline
\end{tabular}


experiment is enforced (see Ref. 5, Fig. 3, p. 852). The internal initial temperature is set to be $T_{i}=\left(T_{h}+T_{c}\right) / 2=303.15 \mathrm{~K}\left(30^{\circ} \mathrm{C}\right)$, the average between hot and cold walls temperature.

The validation of the thermal coupling mechanism involves the two horizontal conductive sheets and the insulator blocks. The initial and boundary conditions are illustrated in Fig. 1. Again, fluid starts from rest, a non-slip condition is applied at the fluid boundaries, and the initial temperature of the fluid is $T_{i}$. Solid media are also set at the initial temperature $T_{i}$, that is, the room temperature specified in Ref. 5.

As regards boundary conditions for the temperature field, at the interfaces between fluidconductors and conductors-insulators, the conjugate heat transfer mechanism is activated. On the left and right sides of the horizontal conducting sheets and insulating blocks, we set a constant temperature, equal to that of the adjacent isothermal wall. This latter choice is justified by the experimental apparatus scheme reported on Ref. 5 (see Fig. 2 on p. 851), where it appears that the horizontal solid blocks are in contact with the isothermal vertical walls. The external faces of insulating blocks are maintained at room temperature $T_{i}$.

For both simulations, periodic conditions are set along the spanwise direction for all variables. The spanwise extension of the domain is long enough to allow development of fully developed turbulence. Specifically, the analysis of correlation functions of velocity and temperature along the spanwise direction (not reported) has shown a complete decay in less than half domain size (see the work of Moin and Mahesh ${ }^{29}$ for a discussion).

Additional analysis (for the case without the CHT) on the mesh and time step dependency, and on the quantification of the unresolved turbulent scales parametrized by the SGS model, is in the Appendix.

\section{THE FLOW FIELD WITHOUT CHT}

Here, we show results of simulations for the fluid domain. After a statistical steady state has been reached, the averaging time to obtain the first and second order statistics corresponds roughly to three turnover times of the main cavity vortex.

\section{A. The mean field}

Fig. 2 shows the non-dimensional mean vertical velocity and temperature over lines, close to the hot wall, at three significant heights: $y / L=0.2,0.5,0.8$.

Vertical velocity profiles, in Fig. 2(a), are in a general good agreement with reference experimental and numerical results, except for the standard Smagorinsky model. Our dynamic Lagrangian model reproduces the decrease of the vertical velocity far from the wall better than the other models. On the other hand, the quality of the results obtained with the standard Smagorinsky model is definitively not good. This has to be attributed to the model itself which is not able to adapt to the local features of the flow field.

Temperature profiles, shown in Fig. 2(b), are quite well reproduced by all models at the intermediate height where comparison with experimental data is possible; some small discrepancies are present in the outer region where radiation effects, not modeled by all numerical simulations, may be present (for a discussion see Ref. 10). At the other locations, all models give results very close to each other.

Fig. 3(a) shows the distribution of mean non-dimensional horizontal velocity component along a vertical centerline $(x / L=0.5)$. Experimental data are not available for this quantity. The three numerical results share the qualitatively behavior. Our simulation gives results very similar to those of Peng and Davidson, ${ }^{11}$ who performed LES with a dynamic Lagrangian SGS model. On the contrary, the standard Smagorinsky model strongly over-predicts the peak velocity in the boundary layer close to the wall.

Comparison among temperature vertical profiles are depicted in Fig. 3(b). All numerical results practically collapse over the experimental data. The peak of temperature in the thermal boundary layer is just slightly over-predicted by the LES of Ref. 11 . 


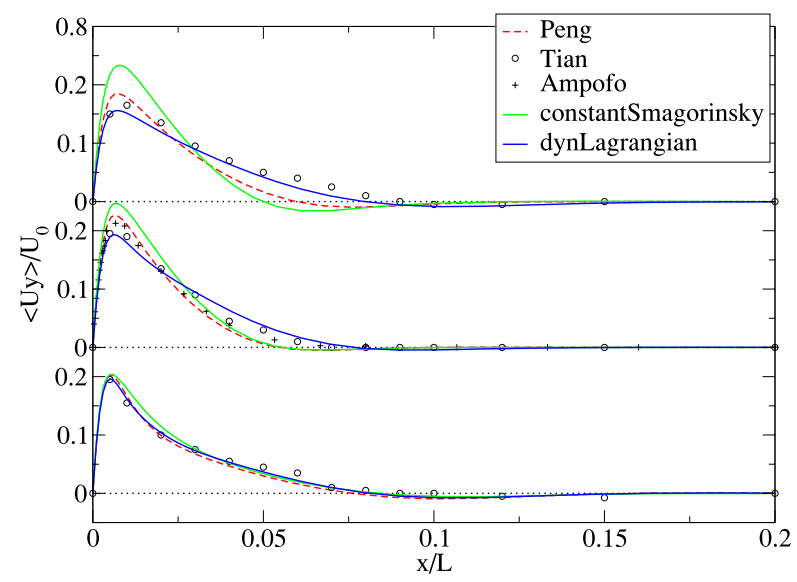

(a)

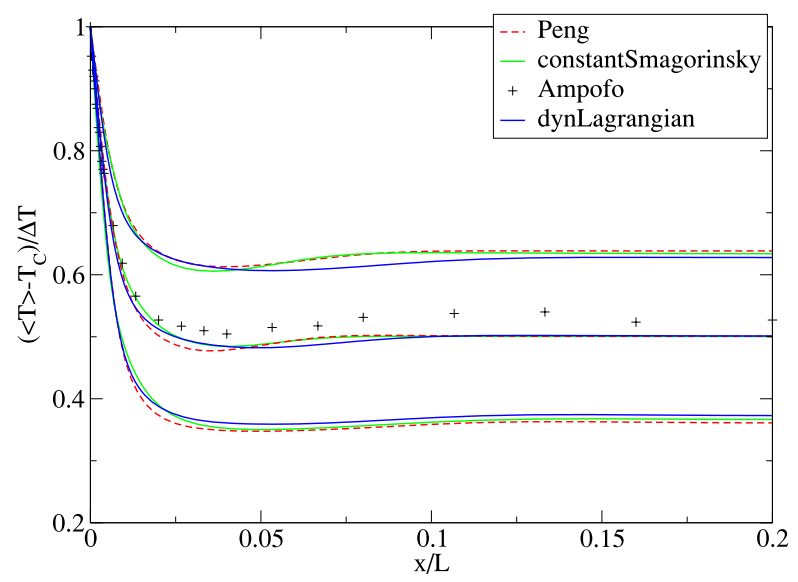

(b)

FIG. 2. Comparison of mean quantities at three different heights along the cavity: $y / L=0.2,0.5,0.8$. Dashed line, LES by Ref. 11; circles, experiment by Ref. 5; crosses, experiments by Ref. 7; and solid light green line, simulation with standard Smagorinsky model; solid dark blue line, simulation with dynamic Lagrangian model. (a) Non-dimensional vertical velocity. (b) Non-dimensional temperature.

Fig. 4(a) shows the streamlines of the mean velocity field. They are in a good qualitative agreement with those depicted in Ref. 11 (see their Fig. 4(a) at p. 327). The motion mainly develops close to the walls through the generation of a main circulation and hence, of four boundary layers. They are narrow close to the two vertical walls, whereas they are thicker at the two horizontal walls. This is expected because the motion is ruled by buoyancy effects arising mainly close to the vertical walls. Low-speed recirculation regions, elongated in the horizontal direction, develop within the core region.

Isothermals are shown in Fig. 4(b). Anti-symmetry with respect to the diagonals of the cubic cavity is evident; for the sake of simplicity, the discussion is focused just on the upper part of the cavity. Hot air is confined in the upper left corner, as a result of temperature transport by the velocity field along the vertical direction. There, the fluid is cooled and spread over a horizontal region of thickness $r / L \cong 0.1$ near the top wall. The core region, approximately a square delimited by $0.15<x / L<0.85$ and $0.15<y / L<0.85$, is characterized by very small velocities and a stable stratification of the flow.

The mean flow exhibits the presence of small vortexes at the top-left/bottom-right corners (Fig. 5). At these corners, the fluid vertical velocity is high because of the buoyancy effects generated by the lateral hot/cold walls, thus generating recirculations and high-speed boundary layers 


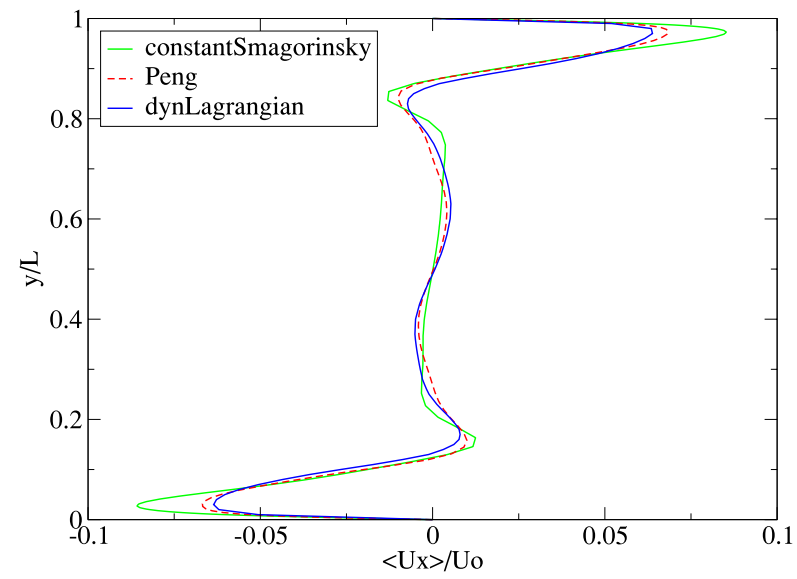

(a)

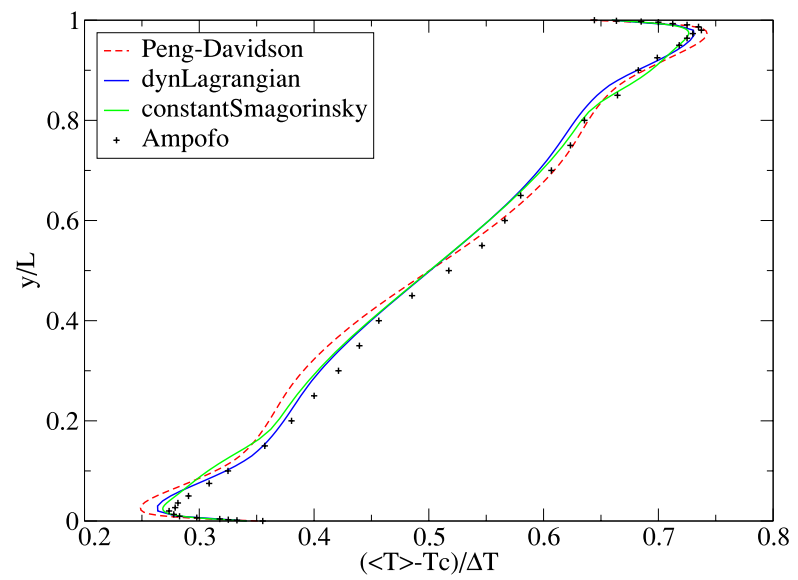

(b)

FIG. 3. Comparison of mean quantities at the vertical centerline $x=0.5 \mathrm{~L}$. Dashed line, LES by Ref. 11 ; crosses, experiments by Ref. 7; solid light green line, simulation with standard Smagorinsky model; and solid dark blue line, simulation with dynamic Lagrangian model. (a) Non-dimensional horizontal velocity. (b) Non-dimensional temperature.

at the horizontal top/bottom walls. These boundary layers, while developing toward the right/left direction loose momentum and the velocity decreases before deviating downward/upward due to buoyancy generated by the cold/hot lateral wall. At the two horizontal walls, typical horizontal convection occurs. This drives the flow from the cold toward the hot region at the bottom wall, and from the hot toward the cold region at the top wall.

The corner vortex dimension is in good agreement with that reported in Ref. 11: the separation point is located at $x_{1} / L=0.9832 \pm 0.0003$ on the top horizontal boundary. A second, smaller and weaker vortex appears very close to the corner; its separation point is located at $x_{2} / L=$ $0.9967 \pm 0.0003$ and this structure is mostly driven by the main vortex surrounding it.

\section{B. Second order statistics}

The second order statistics are now discussed. Fig. 6 presents the RMS of quantities at the same three vertical levels as in Fig. 3.

Overall, the agreement with experimental data is satisfactory. Our results with dynamic Lagrangian model are similar to those of Ref. 11 in which a dynamic SGS model was adopted. 


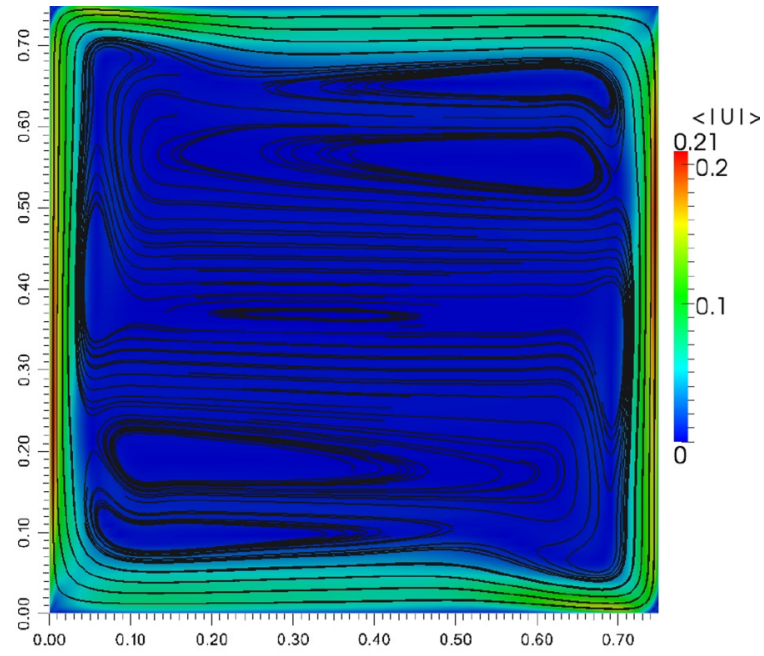

(a)

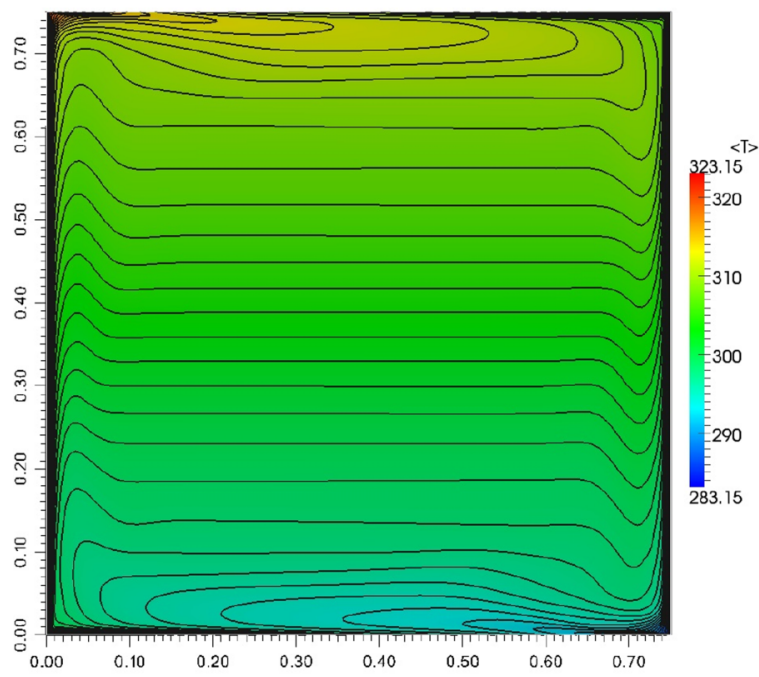

(b)

FIG. 4. Mean flow and thermal field. (a) Streamlines of mean flow $\langle\mathbf{u}\rangle$. (b) Contour plot of the mean temperature field $\langle T\rangle$.

The profiles of temperature root-mean square do not improve with respect to previous simulations. However, the discrepancies, wherever present, do not affect the general feature of the flow and thermal fields.

\section{SIMULATION WITH CONJUGATE HEAT TRANSFER}

The validation of the heat transfer model is performed looking at the temperature profile development.

The main concern in the analysis of fully developed temperature distribution in a solid body regards the diffusion time scale related to geometric features and boundary conditions that can largely exceed the adjustment time of the flow.

Moreover, the implementation of thermal coupling by itself increases the computational cost and may require a small computational time step, depending on the physical characteristics of the 


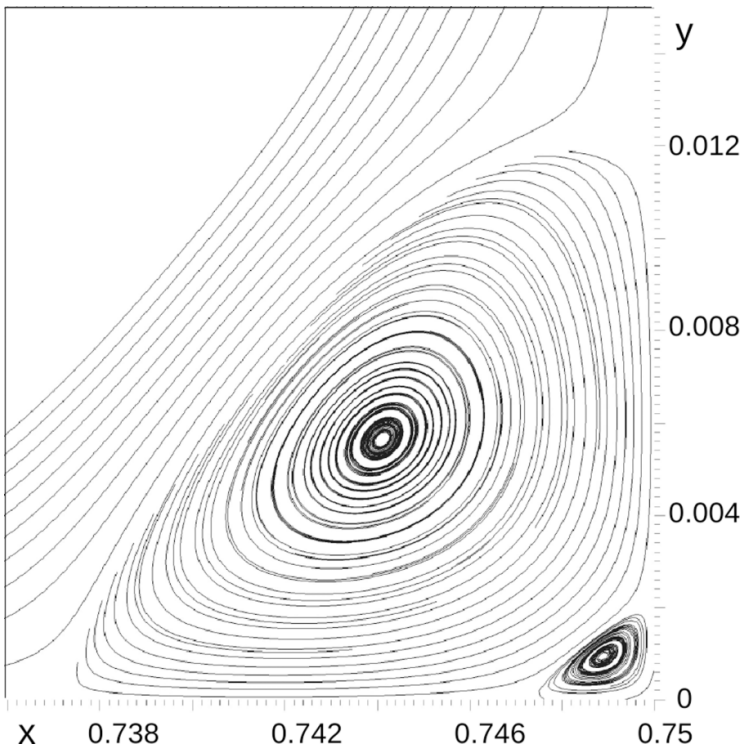

FIG. 5. Mean flow, streamlines at the right-bottom corner neighboring the cold wall.

solid material. Preliminary simulations have shown that an insulator-type material needs smaller time steps than a conductor-type one, thus increasing the computational time required to reach a statistical steady state. Under these conditions, the simulation may become extremely expensive even for a simple configuration.

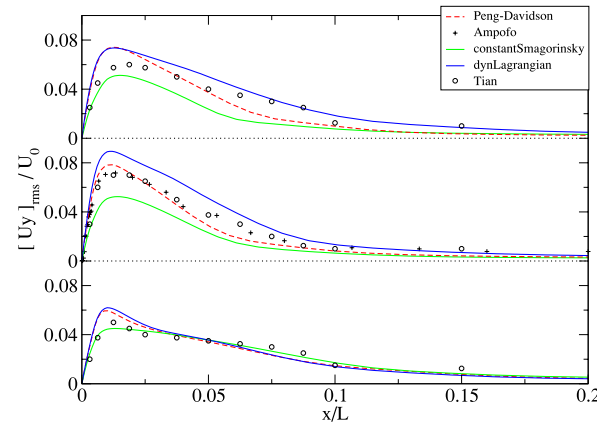

(a)

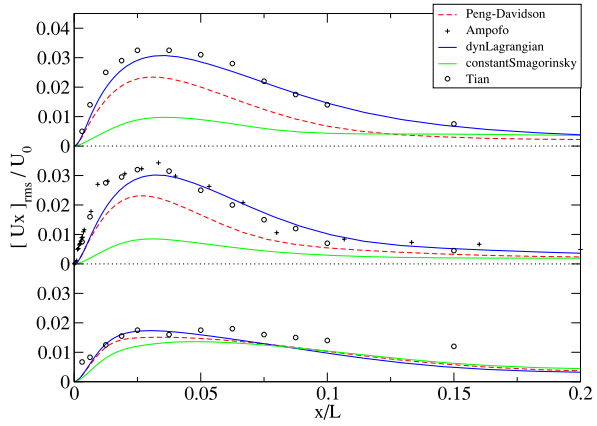

(b)

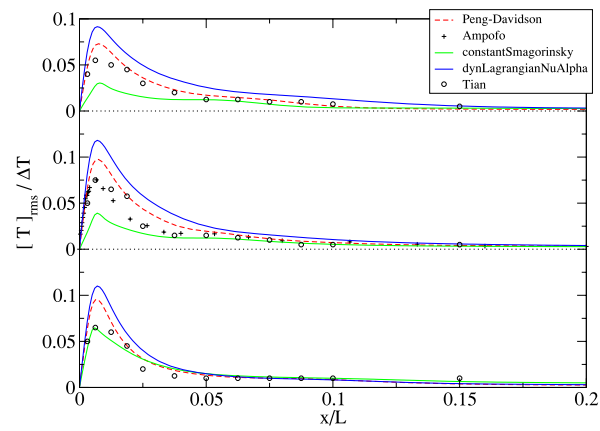

(c)

FIG. 6. Root-mean square of flow quantities near the hot wall, at three levels $y / L=0.2,0.5,0.8$ from bottom to the top. Dashed line, LES by Ref. 11; circles, experiment by Ref. 5; crosses, experiments by Ref. 7; solid light green line, simulation with standard Smagorinsky model; and solid dark blue line, simulation with dynamic Lagrangian model. (a) Non-dimensional vertical velocity RMS. (b) Non-dimensional horizontal velocity RMS. (c) Non-dimensional temperature RMS. 
In this context, a shortcut is introduced to obtain the steady state temperature profile of solid boundaries. As discussed in Sec. III, the time required to completely diffuse temperature within the horizontal solid medium can be estimated as

$$
t \sim \frac{\rho C_{p} L^{2}}{k},
$$

where $L$ is the characteristic length of the solid body. If we artificially decrease the specific heat capacity $C_{p}$, we will decrease also the time required to reach the stable temperature profile. From a physical point of view, this means that the material is able to conduct temperature faster. From a mathematical side, this choice does not influence the heat transfer mechanism, because Equations (6) and (7) do not involve the $C_{p}$ and allows reaching the correct stationary profile. Indeed Equation (5) for steady state reduces to the Laplace equation,

$$
\nabla^{2} T=0
$$

that is independent from specific heat capacity. Thus, the solution is determined only by boundaries conditions.

Therefore, in our simulation, we use the following temperature advancement strategy:

1. First approximation (APP) — simulation with real-medium parameters is run till the fluid part is fully developed. The system is not at a steady state because temperature profile on solid conductive boundaries has not converged to a statistical steady state.

2. Drugging period (DRG) - in order to reach thermal equilibrium, the $C_{p}$ of the solid material is decreased by few orders of magnitude and the simulation run again for a period comparable with the reduced characteristic diffusion time. Temperature diffusion process is now much faster and the temperature profile reaches steady state rapidly.

3. Homogenization (HOM) - temperature distribution in the solid medium is averaged in space in presence of homogeneous directions, or for few time steps. This is done in order to eliminate the non-physical thermal fluctuations arising with the decreased specific heat coefficient.

4. Final adjustment (ADJ) - the actual value of $C_{p}$ is restored and simulation restarts. After an initial adjustment transient, the system can be considered to be in a steady state.

With this procedure, the stationary (in a statistical sense) distribution of temperature in the solid body is reached in a reasonable time, virtually accelerating the temperature diffusion process along the direction over which the body is elongated. This strategy may be generalized for cases involving more solid domains interacting among them.

Another approach for the (DRG)-step is to solve Laplace equation (30) instead of diffusion one (5). From a physical point of view, this corresponds to the limit case of $C_{p} \rightarrow 0$; thus, temperature diffuses instantaneously over the solid domain. The steady profile depends only on the instantaneous interfacial heat flux and is established automatically at each computational time step. This alternative method has been found to lead to numerical instabilities, caused probably by the sharp difference of temperature at the interface. More tests are required to address this problem.

\section{A. Temperature profile within mild steel walls}

The validation of thermal coupling mechanism is here discussed. We refer to Sec. IV D for the description of general settings used.

A first CHT simulation is run to analyze the interaction between the physical elements present in the experiment by Tian and Karayiannis: ${ }^{5}$ fluid is in contact with the horizontal mild steel sheets that, in turn, are in contact with polystyrene elements as shown in Fig. 1.

The characteristic diffusion time for the mild steel sheets is $\tau=4.8 \times 10^{4} \mathrm{~s}$ (corresponding to $13 \mathrm{~h}$ ), obtained using the parameters reported in Table II and considering the sheet length $L=0.75 \mathrm{~m}$. When the advancement strategy is applied, the specific heat capacity is decreased by three orders of magnitude while keeping unchanged $C_{p}$ of insulating bricks of polystyrene. Thus, the diffusion time is reduced to about $\tau \sim 50 \mathrm{~s}$. 
A second simulation is then performed: since the presence of the insulator is negligible when the stable temperature profile is reached, polystyrene is replaced by adiabatic condition on the external faces of the horizontal mild steel sheets. This condition mimics the presence of a perfect insulator.

Fig. 7 shows the non-dimensional temperature distribution over an horizontal line at the mid-height of the top sheet, for the two cases. Note that the two horizontal plates (top and bottom) behaves in the same way. In our analysis, we focus on the top one.

If the CHT is replaced by an idealized adiabatic condition, the temperature on the sheet is determined by the left/right isothermal temperatures, resulting into a linear distribution along the horizontal direction, i.e., $\mathrm{x}$-axis. When the CHT is active, the temperature within the solid medium is still dominated by the constant temperature at the tips of the sheet, but its longitudinal distribution is ruled by the fluid-solid and solid-solid interactions.

Temperature at different time, when polystyrene is present, is reported: after a time $t=100 \mathrm{~s}$ of simulation (corresponding to $\Delta t=10^{5} \mathrm{~s}$, or $27 \mathrm{~h}$ of physical time), the temperature profile reaches a quasi-steady configuration qualitatively similar to the experimental profile. Afterwards, the process slows down because temperature stats to diffuse into the insulator. This process is faster at the beginning when the temperature gradient is larger, and it slows down after that the temperature starts to diffuse in the region near the solid-solid interface and the temperature gradient becomes smaller and smaller.

The simulation is interrupted before reaching the asymptotic regime, because on one side, it takes a huge amount of time (even if the $C_{p}$-decreased strategy is applied) and, on the other side, the steady distribution on the polystyrene depends on the boundaries conditions on its external part only, which are not specified in the description of the experiment. Therefore, the exact experiment configuration cannot be reproduced.

Nevertheless, the simulation exhibits a transitional behavior that at a certain time fits the experimental profile. This may suggest that the experimental profile is still in a transitional phase, and a much longer time would have been requested to obtain the asymptotic profile. In fact, the characteristic diffusion time for the polystyrene is $\tau=3.6 \times 10^{5} \mathrm{~s}$, when the characteristic length is $L=0.75 \mathrm{~m}$, the time required to reach the asymptotic temperature profile in the insulator is of the order of days. The reference paper ${ }^{5}$ does not provide information about the time elapsed from the start of the experiment. However, the authors reported that an incense smoke was used as seeding, and that this smoke lasted in the air cavity for more that $24 \mathrm{~h}$. Thus, assuming an experimental elapsed time of the order of one day, we may argue that it was substantially less than the polystyrene diffusion time, which is of the order of 4 days.

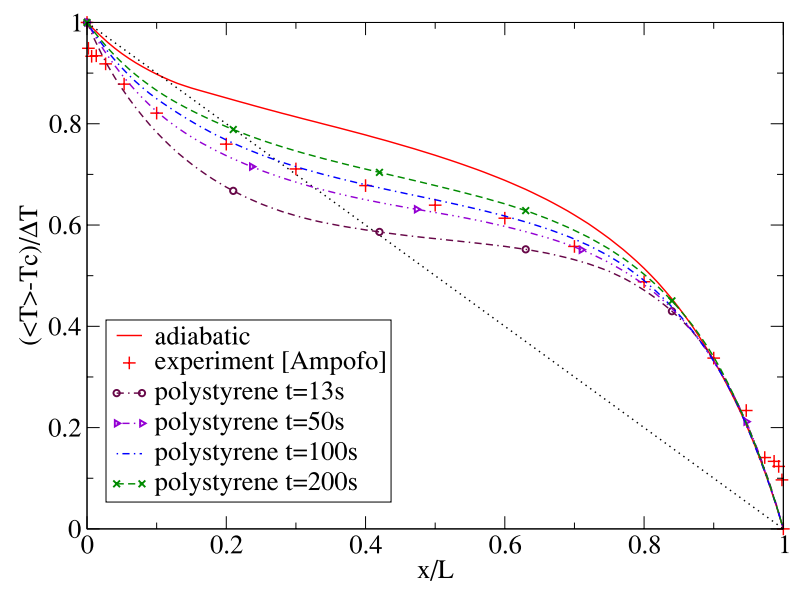

FIG. 7. Temperature distribution over center-line of the top steel sheet. Comparison between experiment data (labeled experiment), simulation with the polystyrene insulator at different times (labeled polystyrene), and a perfect insulator at steady state (labeled adiabatic). 
On the contrary, the case with ideal insulator (adiabatic condition) reaches a steady configuration which appears quantitative different from the experimental profile. This is expected since polystyrene is not a perfect insulator.

To summarize, the analysis carried out in this section shows that the implementation of CHT is able to realistically reproduce the time evolution of a physical process where heat transfer between solid and fluid media is involved. Also, the four-step procedure herein developed allows to obtain the realistic behavior with an acceptable computational cost. Third, a simplified adiabatic condition might be not able to reproduce correctly experimental data, since sometimes the asymptotic condition is hardly reachable in physical experiments.

\section{ANALYSIS OF THERMO-FLUID DYNAMICS INTERACTION USING DIFFERENT MATERIALS}

In this section, we study the influence of conductive walls on the fluid flow. The temperature profile on the horizontal solid sheets is greatly affected by the thermal characteristics of the medium, which, in turn, influences the buoyancy-driven flow.

Once we assign the physical properties of the fluid and the geometry of the case, the behavior of solid boundaries depends on few main parameters:

- the volumetric heat capacity $\rho C_{p}$ represents the thermal inertia of the solid, namely, the tendency to maintain its own temperature profile against the temperature fluctuations of the fluid. Hence, it is more related to the temperature distribution within the solid media;

- the thermal conductivity $k$, links to the heat transfer at the interface, so also to the capability of the materials to modify the fluid temperature. It qualitatively determines the general fluid motion and it rules the interfacial heat flux.

These two quantities are not completely independent on each other in a real medium: usually, a thermal insulator has a low conductivity and low heat capacity, while a conductor has high conductivity and capacity (i.e., the case of metals). Table II reports the thermal properties of some common materials, while Fig. 8 shows the distribution of these materials with respect to their own properties. Materials with high conductivity and low capacity are difficult to find in nature. However, the rapid development of materials science might lead to the production of materials with these characteristics; for this reason, we also consider a virtual material belonging to this group, called Neosyle. ${ }^{2}$

TABLE II. Thermal physical properties of some materials expressed in the International System of Units. Data from Ref. 30, except for those labeled with * that are taken from Ref. 20.

\begin{tabular}{lcrcc}
\hline \hline $\begin{array}{l}\text { Material } \\
\text { units }\end{array}$ & $\begin{array}{c}C_{p} \\
\mathrm{~J} /(\mathrm{kg} \mathrm{K})\end{array}$ & $\begin{array}{c}\rho \\
\mathrm{kg} / \mathrm{m}^{3}\end{array}$ & $\begin{array}{c}k \\
\mathrm{~W} /(\mathrm{m} \mathrm{K})\end{array}$ & $\begin{array}{r}\rho C_{p} \times 10^{-3} \\
\mathrm{~J} /\left(\mathrm{m}^{3} \mathrm{~K}\right)\end{array}$ \\
\hline Polystyrene & 1300 & 15 & 0.03 & 19.5 \\
Glass wool & 675 & 52 & 0.04 & 35 \\
Charcoal & 840 & 240 & 0.05 & 202 \\
PVC* & 900 & 1300 & 0.19 & 1170 \\
Gypsum & 1080 & 1200 & 0.43 & 1296 \\
Concrete stone & 653 & 2300 & 0.93 & 1502 \\
Sandstone* & 920 & 2300 & 1.70 & 2116 \\
Lead & 1296 & 11300 & 35.80 & 14644 \\
Steel (mild) & 500 & 7830 & 45.30 & 3915 \\
Graphite & 670 & 1870 & 130.00 & 1253 \\
Magnesium & 1000 & 1730 & 160.00 & 1730 \\
Copper & 390 & 8910 & 393.00 & 3475 \\
Neosyle & $\ldots$ & $\ldots$ & 100 & 100 \\
\hline \hline
\end{tabular}




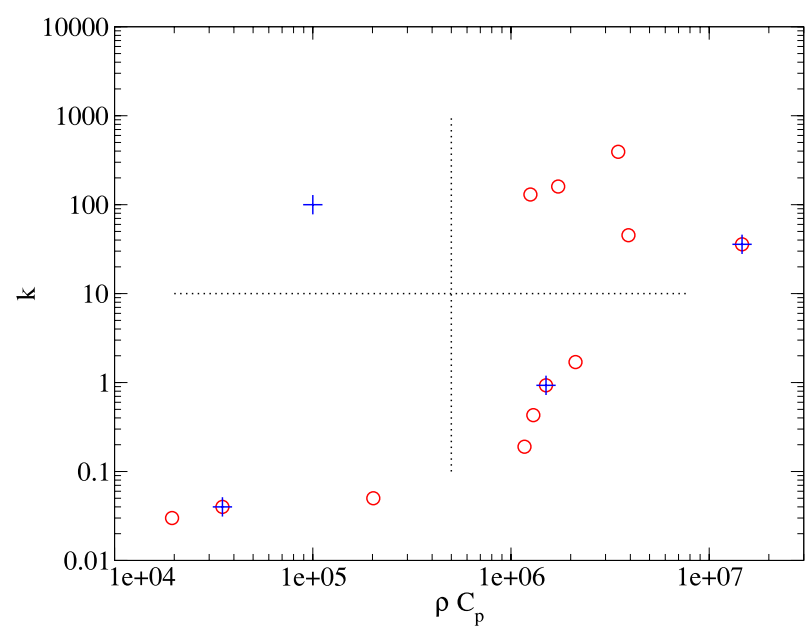

FIG. 8. Relation between thermal conductivity and the specific heat: the circles represent materials of Table II; the pluses represent materials used in simulations.

The following simulations investigate the effect of different materials composing the horizontal walls on the development of the thermo-fluid dynamic field. Five simulations are performed: one with a perfect insulator, the others reproducing the cases shown in Table III, representing the four possible combinations of high/low conductivity and high/low thermal inertia. As already mentioned, Neosyle does not exist in nature, but it is taken as representative of the category with relatively high thermal conductivity and relatively small specific heat, arbitrary chosen as $k=10^{2} \mathrm{~kg} / \mathrm{m}^{3}$ and $\rho C_{p}=10^{5} \mathrm{~J} /(\mathrm{kg} \mathrm{K})$, respectively.

\section{A. Simulations settings and procedure}

First, we run the case with perfect insulator boundaries, where the coupling condition at the solid-fluid interface is replaced by the adiabatic condition.

In order to reduce the computational time, the simulation is initialized using the fluid flow final configuration obtained by the validation case of thermal-coupling, reported in Sec. VI A. The simulation is run until reaching a statistically steady state.

For cases with real horizontal solid sheets, the external insulator blocks present in the experimental apparatus are replaced by an adiabatic condition at the external boundaries of the horizontal sheets; fixed temperature is set at the tips (coherent with the contiguous vertical isotherm boundaries of the fluid domain); CHT is considered during the evolution time of the process at the horizontal solid-fluid interface.

TABLE III. Solid boundaries characteristics. Neosyle is a material with $k=10^{2} \mathrm{~kg} / \mathrm{m}^{3}$ and $\rho C_{p}=10^{5} \mathrm{~J} /(\mathrm{kg} \mathrm{K})$ that does not exist in nature.

\begin{tabular}{ccc}
\hline \hline & $\rho C_{p}<10^{6}$ & $\rho C_{p}>10^{6}$ \\
\hline & Neosyle: & Lead: \\
$\tau=5.6 \times 10^{2}$, & $\tau=2.3 \times 10^{5}$, \\
$k>10$ & $\alpha=1.0 \times 10^{-3}$, & $\alpha=2.45 \times 10^{-6}$, \\
& $T A R \cong 1.75 \times 10^{-3}$ & $T A R \cong 2.4 \times 10^{-4}$ \\
& Glass wool: & Concrete: \\
& $\tau=3.65 \times 10^{5}$, & $\tau=0.9 \times 10^{6}$, \\
$k<10$ & $\alpha=1.54 \times 10^{-6}$, & $\alpha=0.62 \times 10^{-6}$, \\
& $T A R \cong 2.3 \times 10^{-1}$ & $T A R \cong 0.5 \times 10^{-2}$ \\
\hline \hline
\end{tabular}


The initial temperature for solid media is set to be the room temperature $T_{i}=303.15 \mathrm{~K}$, in analogy of validation settings in Sec. IV D. In order to reduce the transient phase, a linear temperature distribution may be imposed along the $x$-direction. This is the asymptotic distribution in case of absence of solid-fluid interaction. Here, we have chosen to set an uniform temperature distribution along the solid sheet because it is closer to an initial experimental situation.

Simulations with CHT are carried out following the temperature advancement strategy described in Sec. VI. For the (APP)-step, the final configuration obtained from the perfect insulator is used as a initial field. In the (DRG)-step, $C_{p}$ is decreased by three orders of magnitude, and simulations are run for $\Delta t_{1}=50 \mathrm{~s} \cong 67 t_{0}$ for Neosyle, lead, and concrete. For the glass wool case, the drugging period is reduced to $\Delta t_{1}=20 \mathrm{~s} \cong 27 t_{0}$ since the thermal coupling for strong insulator dramatically increases the computational time.

In the (HOM)-step, temperature is averaged on the homogeneous $z$-direction. In the (ADJ)step, the correct heat capacity is restored and simulations are run again for $\Delta t_{2}=10 \mathrm{~s} \cong 13 t_{0}$, a period long enough to bring to steady state condition the fluid/solid interaction. Afterwards, data are collected for successive $\Delta t_{3}=60 \mathrm{~s} \cong 80 t_{0}$ for the statistical analysis. In this time interval, the systems are considered in a quasi-steady state, i.e., the evolution of temperature within solid media is negligible during $\Delta t_{3}$.

The time simulated in the (DRG)-step is equivalent to a physical time $\Delta t_{\text {phy }} \sim 13 \mathrm{~h}$ for all materials except for glass wool, for which it corresponds to $\Delta t_{p h y} \sim 5 \mathrm{~h}$. The estimation of the physical time can be obtained from the non-dimensional time parameter,

$$
\Pi_{t}=\frac{\Delta t_{1}}{\tau_{\text {red }}}=\frac{\Delta t_{p h y}}{\tau_{p h y}},
$$

where $\tau_{\text {red }}$ is the characteristic time computed with the reduced $C_{p}$ and $\tau_{p h y}$ is the characteristic time of the actual materials. This non-dimensional time must be the same for the systems with actual and reduced specific heat. Notice that $\Pi_{t}$ gives also an estimation of the degree of thermal development of solid media: $\Pi_{t}>1$, temperature has reached a steady profile; $\Pi_{t}<1$, temperature is still evolving. Table IV reports the non-dimensional time for our cases.

The interval $\Delta t_{1}$ was observed to be sufficient to reach a stationary distribution of temperature within the solid just for the Neosyle case; it was not long enough for the other materials having larger characteristic diffusion times.

Even if the statistical steadiness for the systems is not reached, the transitional configurations obtained can be representative of applications and experimental apparatus. For this reason, we retained all cases, limiting our attention just to the differences arising in transitional configurations, sharing the same physical time.

However, Neosyle and the perfect insulator deserve particular attention: they represent fully developed systems for the categories of conductors and insulators, respectively.

TABLE IV. Geometrical characteristics of the recirculation bubbles on the top horizontal plate and non-dimensional time parameter. Columns: first, location of separation point; second, location of reattachment point; third, maximum distance from the plate $(h)$ of the bubble; and fourth, non-dimensional time $\Pi_{t}=\Delta t_{1} / \tau_{\text {red }}$, where $\Delta t_{1}$ is the time simulated in (DRG)-step and $\tau_{\text {red }}$ is the characteristic time computed with the reduced $C_{p}$ coefficient. Geometrical estimation from the stream line plots; absolute error $e_{a b s}= \pm 0.003$.

\begin{tabular}{lcccc}
\hline \hline & $\begin{array}{c}\text { Separation point } \\
{[\mathrm{x} / \mathrm{L}]}\end{array}$ & $\begin{array}{c}\text { Reattachment } \\
\text { point }[\mathrm{x} / \mathrm{L}]\end{array}$ & $\begin{array}{c}\text { Height } \\
{[\mathrm{h} / \mathrm{L}]}\end{array}$ & $\Pi_{t}[-]$ \\
\hline Neosyle & 0.167 & 0.267 & 0.0267 & 90 \\
Lead & 0.120 & 0.207 & 0.0267 & 0.22 \\
Concrete & 0.127 & 0.173 & 0.0067 & 0.05 \\
Glass wool & 0.087 & 0.153 & 0.0067 & 0.05 \\
Perfect insulator & $\ldots$ & $\ldots$ & $\ldots$ & $\ldots$ \\
\hline \hline
\end{tabular}




\section{B. First order flow statistics}

In the following, we classify under the general definition of insulators the materials with low $k$ (glass wool and concrete) and conductors those with large $k$ (Neosyle and lead).

Fig. 10 shows the streamlines of the mean velocity field for the five cases. The most interesting features are the recirculation regions at the horizontal walls, near the top-left and bottom-right corners, which are absent in our case with perfect insulator. To the knowledge of the authors, previous works ${ }^{11,5,6}$ studying the effects of conductive boundaries in a cavity did not report about this flow feature, which, in fact, is a direct consequence of temperature distribution at the boundaries arising after the solid-fluid thermal interaction.

The system is antisymmetric respect to the two diagonals of the domain. For this reason, we focus the following analysis only in the top-left region.

As a general feature, hot air blows up from the vertical left wall and impacts the cavity ceiling, then it bounces off and the original upward vertical motion deviates along two directions: a prevalent horizontal one and a weaker, downstream, vertical one. The deflection of the main stream creates two low-speed regions highlighted in Fig. 10: one is on the left side of the impact region where the corner vortex appears; the other is on its right side close to the horizontal wall.

In the case of the perfect insulator, the velocity is not large enough to generate flow separation; in the other cases, the wall conducts heat and tends to warm up the fluid near the left wall and to cool it near the right wall. Therefore, a thin layer of colder fluid travels towards the right wall after being deflected, giving rise to a vertical buoyancy force that pushes the flow closer to the horizontal plate. This causes an increase of horizontal velocity, thanks to the reduction of the flow section. The increased velocity produces flow separation and generates a recirculation bubble of warm fluid. This bubble is found to be larger for conductors since they better cool down the fluid, while it disappears with the perfect insulator.

Fig. 9 clearly shows the role of the CHT on the temperature distribution close to the horizontal top wall. The perfect insulator (i.e., adiabatic conditions) produces an homogeneous distribution of temperature along the horizontal direction. This feature is shared by the other insulators (glass wool and concrete). Moving toward conductors and in particular for Neosyle, the flow temperature decreases from left to right. This happens because the conductor supplies and drains heat, respectively, by the left and right side of upper portion of the cavity. In all cases, the core region exhibits a stable thermal stratification: as shown in Sec. VII C, the turbulent fluctuations are almost absent far from the walls.

Fig. 10 depicts the streamlines of the mean flow. The cases other than perfect insulator and Neosyle may suffer from lacking of steadiness. Most of the fluid motion takes place near the boundaries, within a layer $r / L<0.1$, in form of a clockwise circulation driven by the convective processes at the two vertical walls. Successive analysis (see Figs. 12(a) and 13(a)) will show that outside of this region, the magnitude of the velocity drops by more than one order of magnitude. The remaining flow field is characterized by stable stratification and horizontally elongated low-speed vortexes in the upper region of the cavity, below the top boundary layer. The perfect insulator case produces a more uniform distribution of the temperature along the horizontal wall and this reflects onto a more streamlined velocity field. The conductor cases, and specifically the Neosyle, where a statistically steady case has been reached, exhibit two triangularly shaped horizontal vortex structures that are the result of the horizontal temperature gradients already discussed.

In Fig. 11, the presence of the typical corner vortex is shown together with the recirculation bubble. Table IV reports the location of separation and reattachment points and the height of the bubbles, together with the index $\Pi_{t}$ of thermal development of the plates.

Again, we focus on the two cases where statistical steadiness has been reached. The main difference stands in the absence, in the insulator case, of the small horizontal vortex placed at the top wall. This structure is persistent, displaying low-intensity turbulence fluctuations. It is generated from the heat transfer mechanism at the fluid/solid interface, which enhances the vertical velocity of the flow approaching the top horizontal wall. In case of Neosyle, the bubble is at steady condition, while for other materials, is present but still slowly evolving in time. 


$\begin{array}{rr}290 \quad 300^{\langle T\rangle} & 310,320 \\ 283.15 & 323.15\end{array}$

(a)

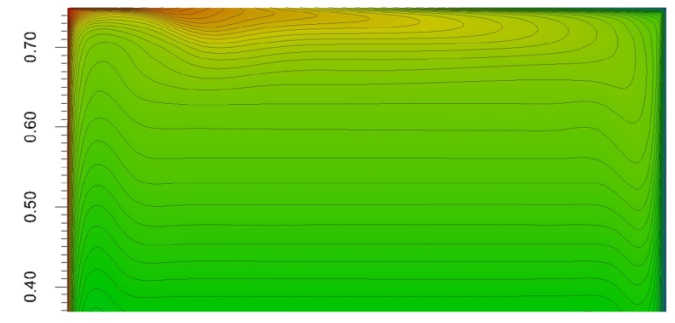

(b)

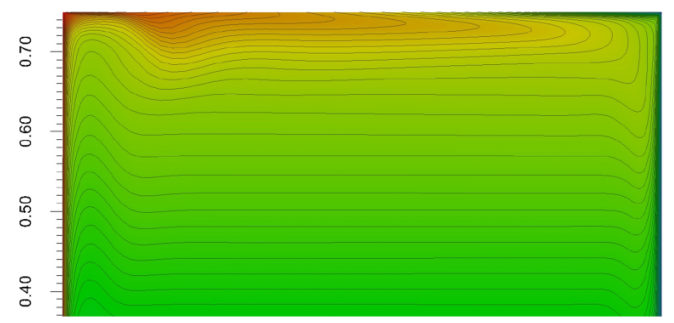

(c)

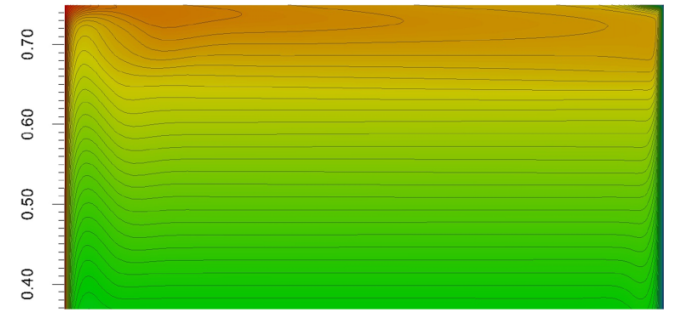

(d)

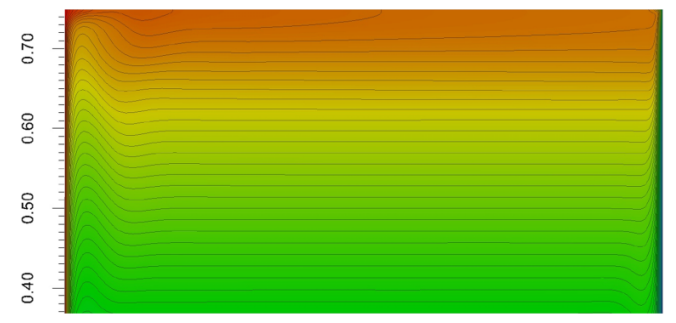

(e)

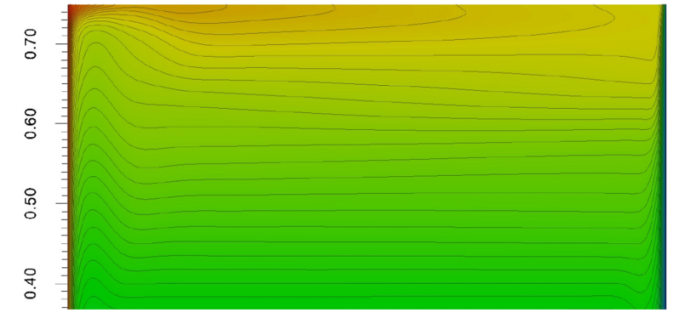

(f)

FIG. 9. Temperature contour plot of the upper half-part of the cavity. (b) Neosyle. (c) Lead. (d) Concrete stone. (e) Glass wool. (f) Perfect insulator. 


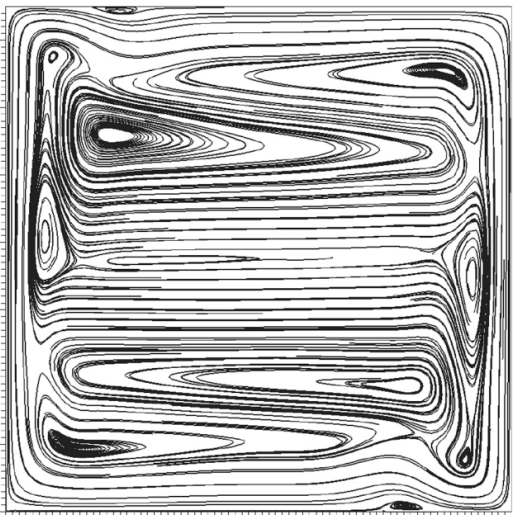

(a)

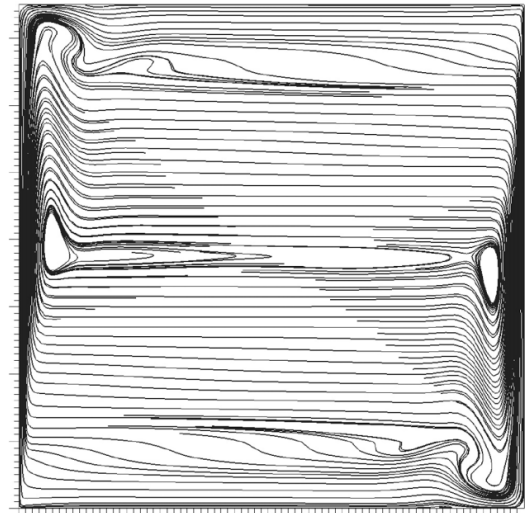

(c)

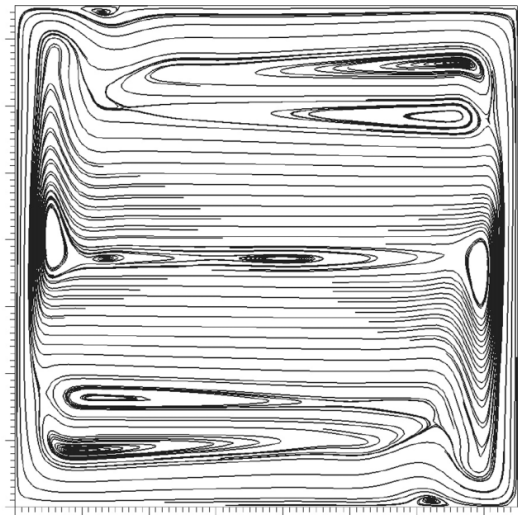

(b)

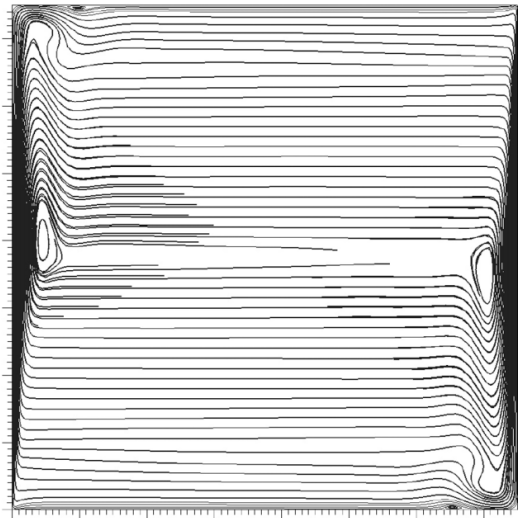

(d)

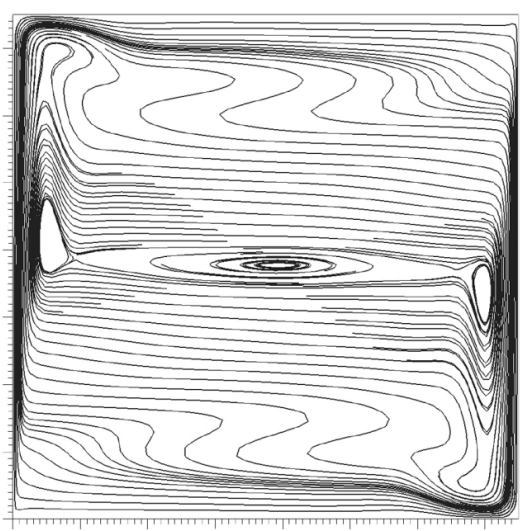

(e)

FIG. 10. Streamlines of $\langle U\rangle / U_{0}$. (a) Neosyle. (b) Lead. (c) Concrete stone. (d) Glass wool. (e) Perfect insulator.

Other minor difference between Neosyle and perfect insulator is that the stable vertically elongated vortex, localized in the center of figure, appears a bit different in shape. For the other cases, this feature is not present on the average, but the instantaneous fields show the occurrence of some unstable vortexes in the same region. At this simulation stage, the turbulence fluctuations rapidly destroy the structure.

Fig. 12 reports the profiles of non-dimensional temperature and vertical velocity at the three heights as in Fig. 2. 


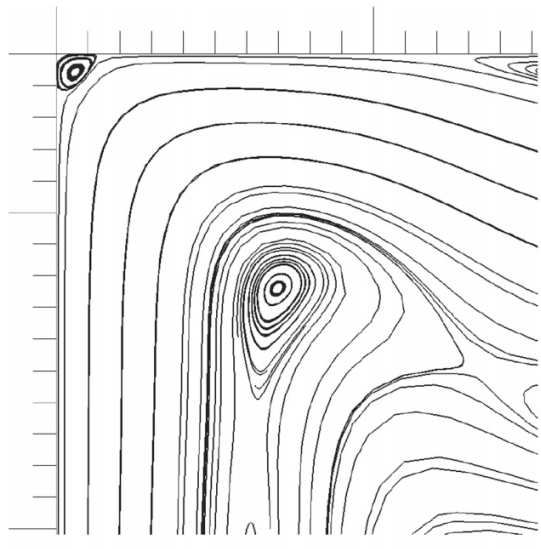

(a)

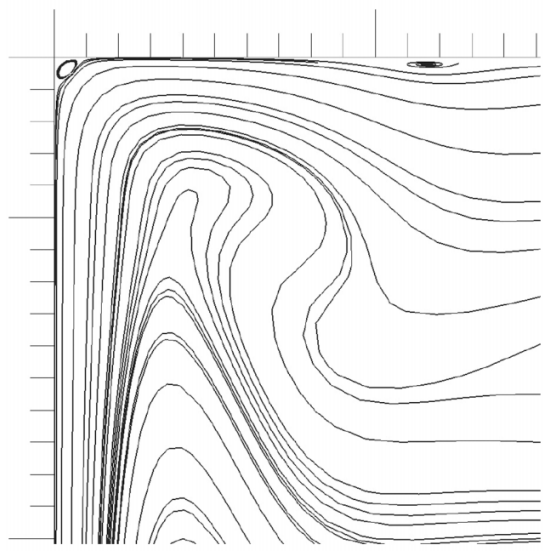

(c)

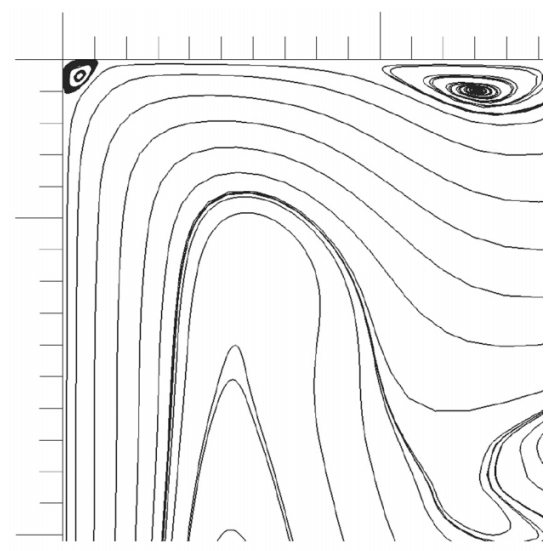

(b)

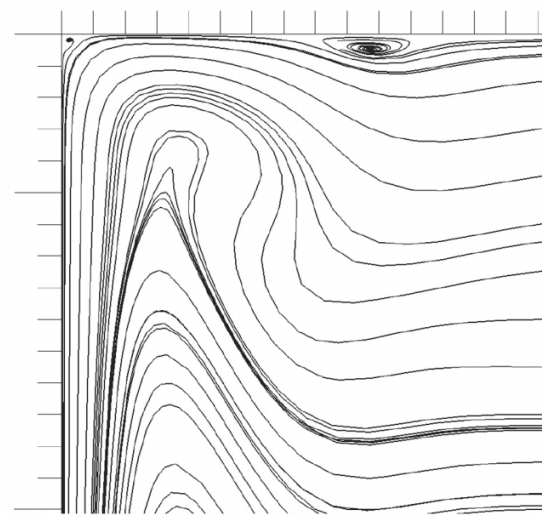

(d)

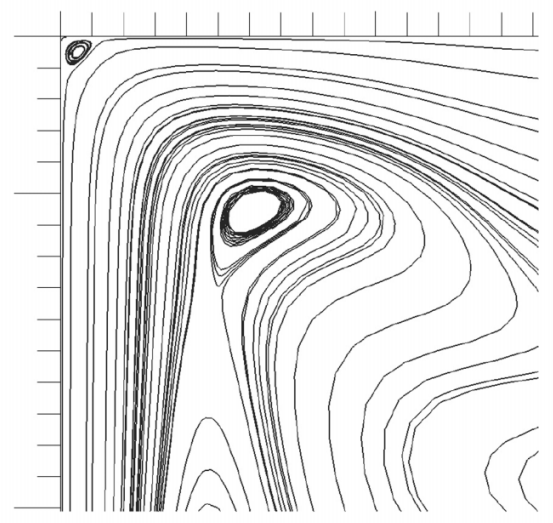

(e)

FIG. 11. Focus on the corner streamlines. The square has an edge of 0.2 L. (a) Neosyle. (b) Lead. (c) Concrete stone. (d) Glass wool. (e) Perfect insulator.

The vertical velocity near the hot wall (Fig. 12(a)) does not exhibit significant differences from case to case at heights $y / L=0.5$, whereas at $y / L=0.8$, vertical velocity is higher for the Neosyle than for the perfect insulator. This difference is even more evident at level $y / L=0.2$. In fact, here air is warmed up not only by the isothermal vertical wall but also by the adjacent conducting floor of the cavity which, in turn, produces a wide region of convective flow. 


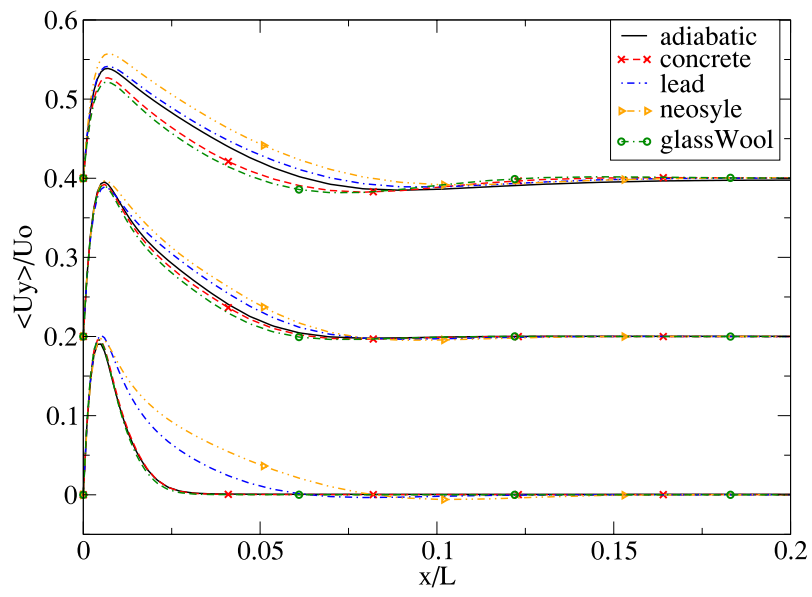

(a)

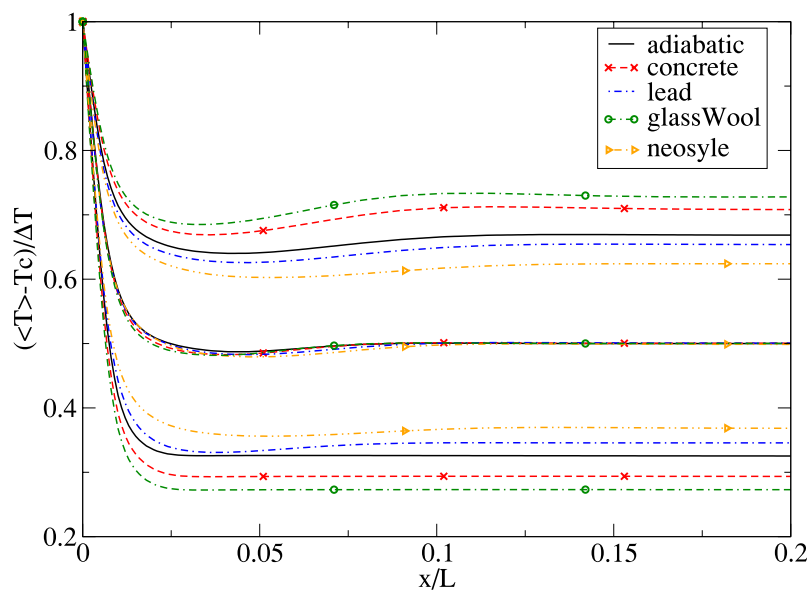

(b)

FIG. 12. Non-dimensional mean vertical velocity and temperature profile close to the hot wall, taken along the three horizontal lines as in Fig. 2. (a) Non-dimensional vertical velocity. (b) Non-dimensional temperature.

The temperature profiles in Fig. 12(b) do not differ among them in the near wall region. However, the temperature in the region farther from the wall is larger in case of conductors rather than for insulators close to the bottom surface $(y / L=0.2)$, whereas the opposite is true at $y / L=0.8$. This follows from the accumulation of high/low temperature occurring close to the top/bottom boundaries in case of insulator media, phenomenon not present when the horizontal plates are made of conducting materials.

Fig. 13 shows non-dimensional mean horizontal velocity and temperature on vertical lines near the top walls, at three significant distances from the hot wall: $x / L=0.2,0.5,0.8$. In case of Neosyle, a more energetic horizontal velocity is observed close to the cavity ceiling. In case of perfect insulator, the velocity decreases more rapidly along the horizontal and this may be related to the horizontal homogenization of the thermal field in the near wall region. The other insulator cases suffer for a transient behavior, the flow field is not completely developed and it is characterized by smaller velocities. Along the line $x / L=0.2$, the velocity peaks at different vertical locations in cases of perfect insulator and Neosyle, correspondingly to the location of the recirculation bubble (see Fig. 11 and Table IV).

The vertical distribution of temperature (Fig. 13(b)) indicates that, in case of Neosyle, the thermal field varies substantially along the horizontal direction close to the top wall, whereas this does not happen for the perfect insulator. This scenario is explained by the fact that conductors 


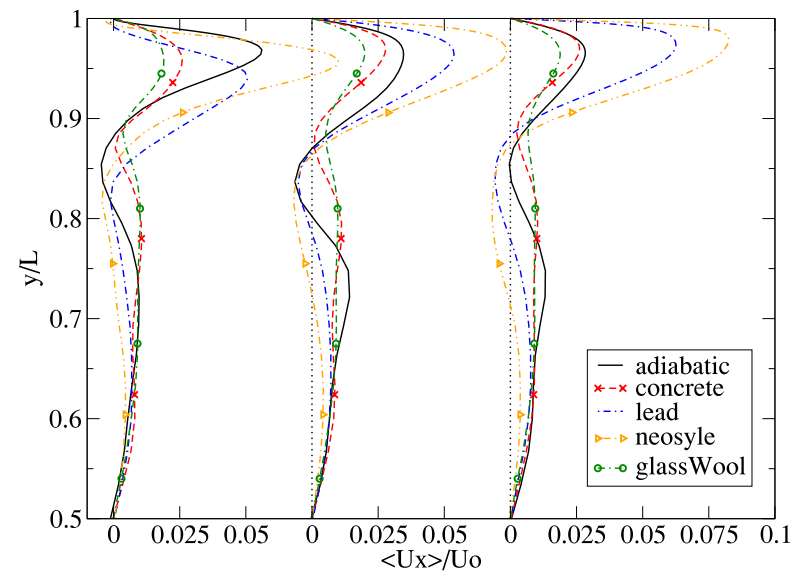

(a)

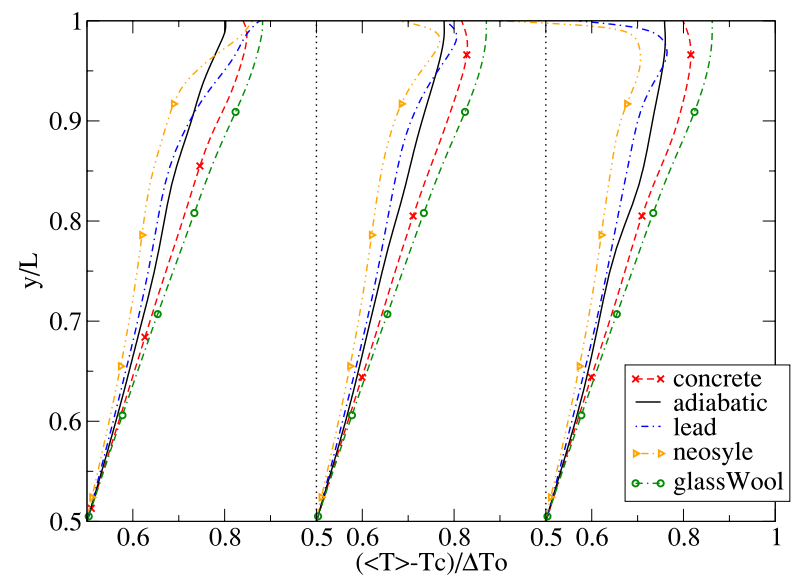

(b)

FIG. 13. Non-dimensional mean horizontal velocity and temperature profile close to the top wall, along three significant vertical lines: $x / L=0.2,0.5,0.8$. (a) Non-dimensional horizontal velocity. (b) Non-dimensional temperature.

supply/drain temperature to/from the fluid region in a not homogeneous way moving downstream along the horizontal wall.

\section{Fluid flow turbulent statistics}

Turbulent statistics near the top and left walls are presented in the following. The core region is characterized by a weak laminar flow: at a distance of $0.2 L$ from the walls $T_{r m s} \sim 0$ and $\mathbf{u}_{r m s} \sim 0$. For this reason, we focus our attention in the near-walls region.

Figs. 14 and 15 show the vertical and horizontal turbulent temperature fluxes. The results can be interpreted via the gradient-diffusion hypothesis (cf. Pope ${ }^{31}$ ): turbulent transport of temperature can be represented as a down-gradient mechanism, that can be modeled by

$$
\left\langle T_{f}^{\prime} u_{i}^{\prime}\right\rangle=-\alpha_{e f f} \frac{\partial T_{f}}{\partial x_{i}},
$$

where $\alpha_{\text {eff }}>0$ is the effective diffusivity. Hence, the temperature gradient has opposite sign with respect to the turbulent fluxes.

Although for the sake of completeness we show results for all cases, here we discuss the difference between Neosyle and the perfect insulator. 

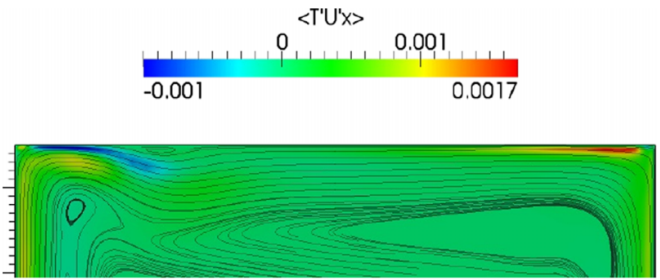

(a)

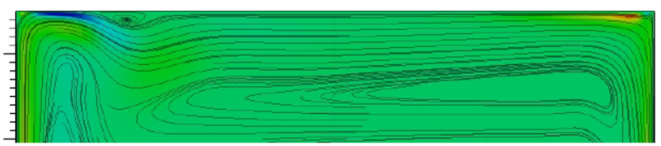

(b)

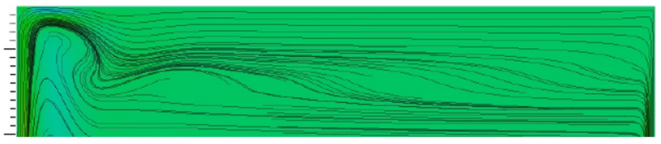

(c)

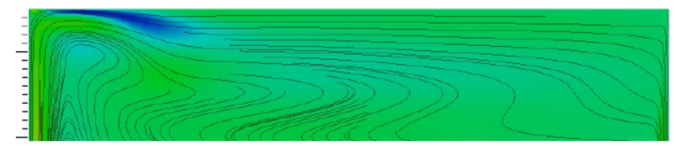

(d)

FIG. 14. Temperature fluxes $\left\langle u_{x}^{\prime} T^{\prime}\right\rangle / U_{0} \Delta T$ near the top horizontal wall. Strips height $0.2 L$, width $1 L$. Streamlines as in Fig. 10 are also reported. There are no significant differences between concrete and glass wool, so the latter is not reported. (a) Neosyle. (b) Lead. (c) Concrete. (d) Perfect insulator.

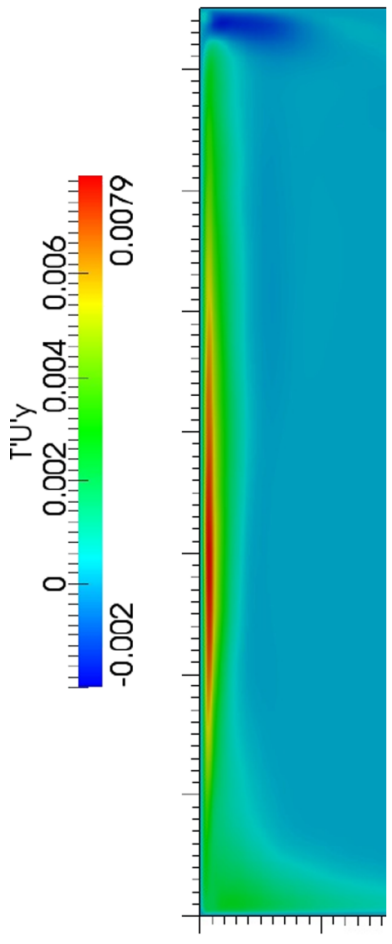

(a) (b)

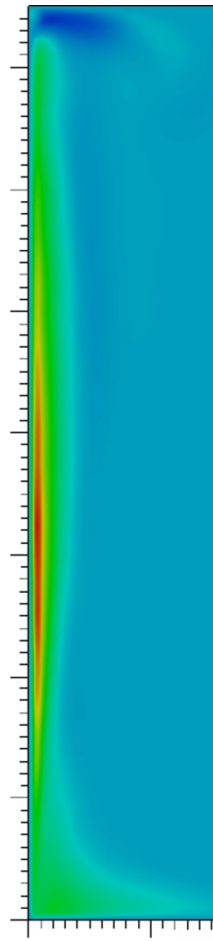

(c)

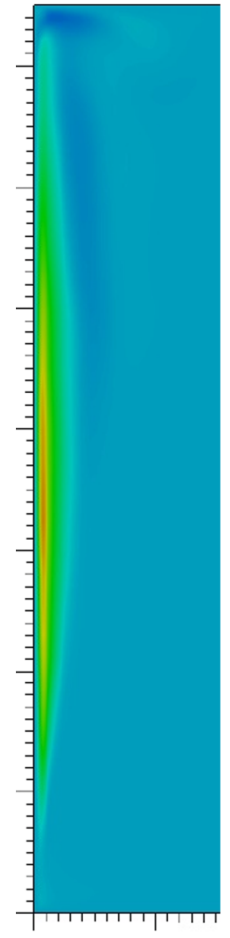

(d)

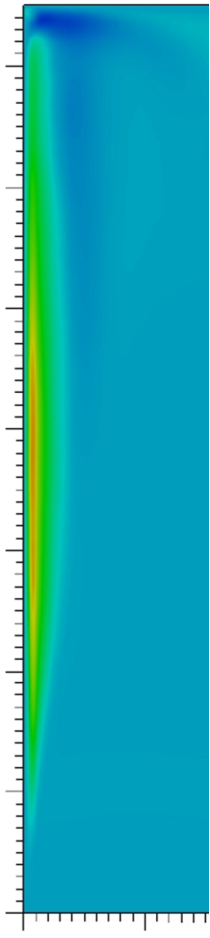

(e)

FIG. 15. Temperature fluxes $\left\langle u_{y}^{\prime} T^{\prime}\right\rangle / U_{0} \Delta T$ near the left vertical wall. Strips height $0.1 L$, width $0.2 L$. There are no significant differences between concrete and glass wool, so the latter is not reported. (b) Neosyle. (c) Lead. (d) Concrete. (e) Insulator. 
Fig. 14 shows the average horizontal turbulent temperature flux in a region $1 \times 0.2 L$ close to the top wall. Also, streamlines are reported for the sake of clarity. A feature common to all cases is the negative spot near the left corner: this corresponds to a positive horizontal turbulent heat flux, that increases the overall horizontal heat convection from the hot wall. The perfect insulator case has a larger negative plume, thanks to the lack of the recirculation bubble, while Neosyle presents a positive spot near the right corner.

In this thin region, close to the horizontal wall, turbulence drives heat from the right to the left lowering the air temperature. This effect is evident in Fig. 13(b) on line $x / L=0.8$, where the fluid temperature of Neosyle case decreases rapidly while approaching the top boundary.

Fig. 15 shows the average vertical turbulent temperature flux on a strip $0.2 \times 1 \mathrm{~L}$ near the vertical hot wall. In all cases, a zone of negative flux near the top corner is present, more intense for conductors than for insulators. In this region, the vertical heat flux is positive.

In the Neosyle case, the cold air layer in the left-bottom corner region triggers a local convection region, which is identified by positive vertical thermal fluxes. The perfect insulator case does not exhibit such a behavior, as expected. The effects of this mechanism reflect also in the less localized vertical velocity profile of the Neosyle case, as shown in Fig. 12(a), at line $y / L=0.2$.

Fig. 16 reports the averaged RMS of temperature, vertical, and horizontal velocity near the horizontal top wall.

In conjunction with the previous plots of heat fluxes, Fig. 16(a) exhibits a significant increase of turbulence for Neosyle, near the right wall. On the other hand, the perfect insulator is characterized by low fluctuations on lines $x / L=0.5,0.8$. Over the same lines, Fig. 16(b) points out that horizontal fluctuations for conductors are localized near the horizontal wall, where unstable stratification triggers turbulence.

In Fig. 16(c), the temperature RMS highlights the main difference between perfect insulator and the real materials. The adiabatic condition allows for higher temperature fluctuation on the wall

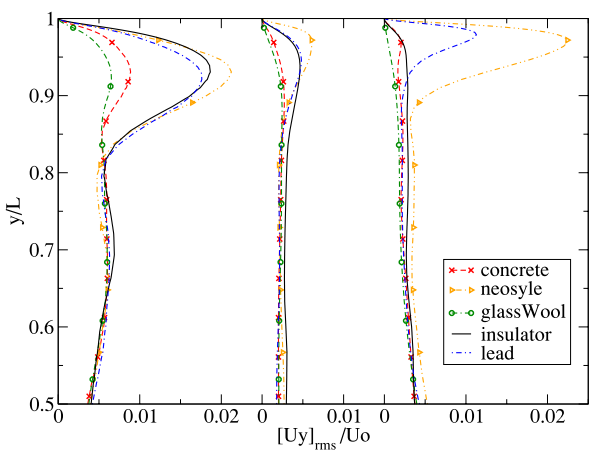

(a)

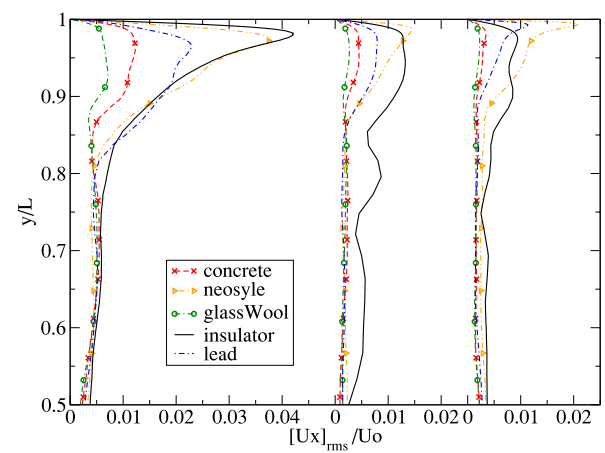

(b)

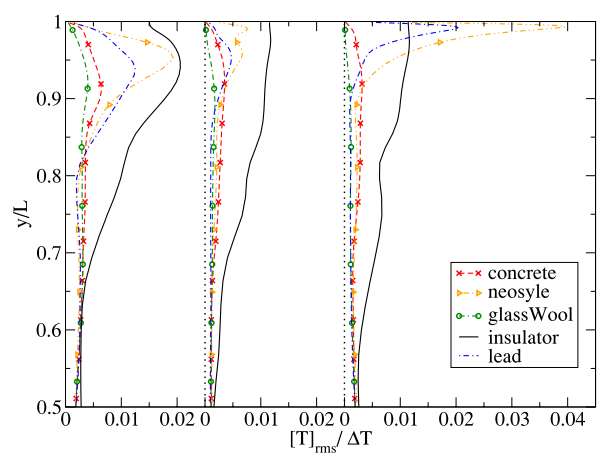

(c)

FIG. 16. Velocity and temperature root-mean square near the top horizontal wall. Plots over vertical lines at the three significant heights as in Fig. 2. (a) Vertical velocity RMS. (b) Horizontal velocity RMS. (c) Temperature RMS. 
that damp thermal gradient and homogenize temperature near the horizontal wall. This eventually inhibits the formation of some flow structures, as the recirculation bubble. For the real materials, fluctuations peak near the horizontal plates while, for the ideal insulator they are scattered on the center region.

\section{Temperature distribution within the solid media}

In presence of fluid-solid coupling, the interfacial heat transfer modifies the temperature distribution in the solid media.

Temperature diffuses in plates along vertical and horizontal direction of propagation, that have two different characteristic time,

$$
\tau_{\text {hor }}=\frac{L^{2}}{\alpha_{s}} \quad \text { and } \quad \tau_{v e r}=\frac{H^{2}}{\alpha_{s}},
$$

where $L=0.75 \mathrm{~m}$ and $H=0.0015 \mathrm{~m}$ are the height and the width of the plates, respectively. Therefore, horizontal diffusion is extremely slower with respect to vertical diffusion, since $\tau_{v e r}=$ $4 \times 10^{-6} \tau_{h o r}$. Due to the high value of the geometrical aspect ratio (thus, independent from the substance properties), the time required to balance the effects of the isothermal edges of the plate and the solid-fluid heat exchange may be huge.

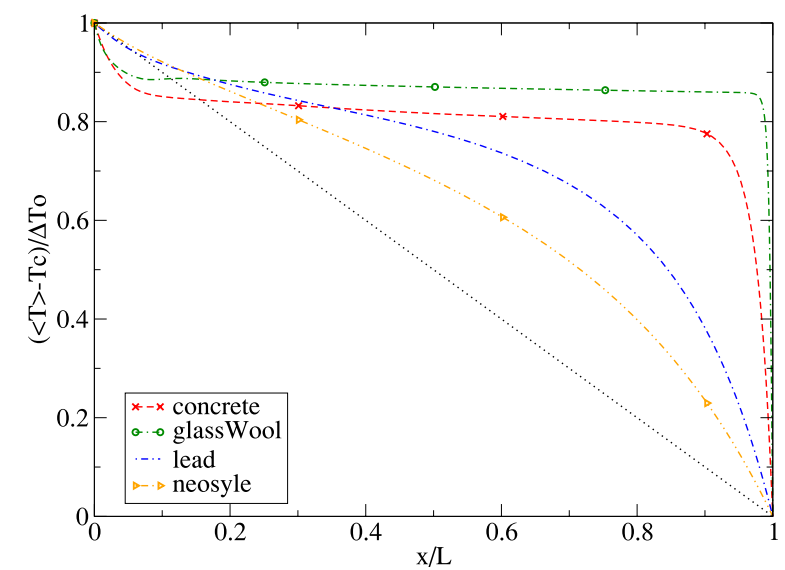

(a)

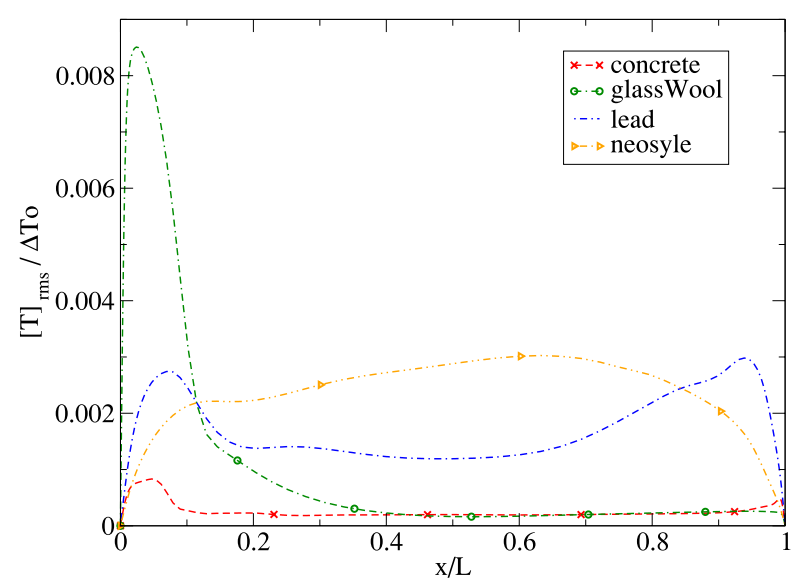

(b)

FIG. 17. Profile among the horizontal center-line of top solid wall. (a) Non-dimensional temperature distribution. (b) Non-dimensional temperature RMS. 
The horizontal characteristic diffusion time of the materials used in the present paper, reported in Table III, results to be far larger than the flow setting time.

Fig. 17(a) shows the temperature profile along a centerline within the solid plate for different materials. The simulations are carried out up to different development stages as it is shown by the non-dimensional time in Table IV. The transitory profiles suggest that, at the early stages, heat transfer from the fluid to the solid medium rapidly increases the internal temperature. This occurs uniformly along the horizontal $x$-direction and is a very fast process since vertical diffusion is very fast. After that the tip effects become increasingly important and the isothermal walls modify the longitudinal temperature distribution: the hot vertical wall warms the horizontal solid medium on the left side, whereas the opposite occurs at the right edge due to the presence of the cold isothermal wall.

The statistical steady temperature profiles within the solid are given by Laplace equation (30) that does not depend on any physical parameter of materials. They are determined only by the heat flux across the solid-fluid interface that is ruled by Equation (7). Indeed, it is expected that materials with similar thermal conductivity tend to develop a similar temperature distribution.

Conductors exhibit an internal temperature profile, on average, higher then the linear profile, reflecting the inhomogeneity in the vertical heat flux along horizontal direction. For insulators the steady state profiles are predicted to have a distribution closer to a linear one, with a lower average temperature with respect to conductors, but this behavior is still not appreciable in our transitory/transitional results.

Fig. 17(b) shows the temperature root-mean square profiles along the horizontal centerline within the top solid medium. The temperature RMS within the solid is nearly constant along the vertical direction, as reported in Fig. 18. This is an effect of the small value of characteristic time for vertical diffusion: the horizontal sheets are thin enough to be strongly influenced by the interfacial fluxes.

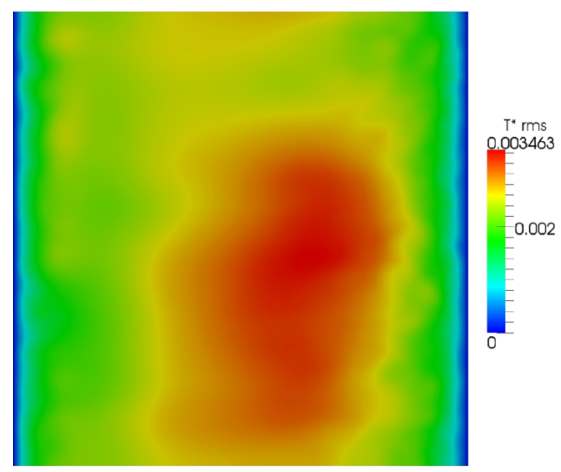

(a)

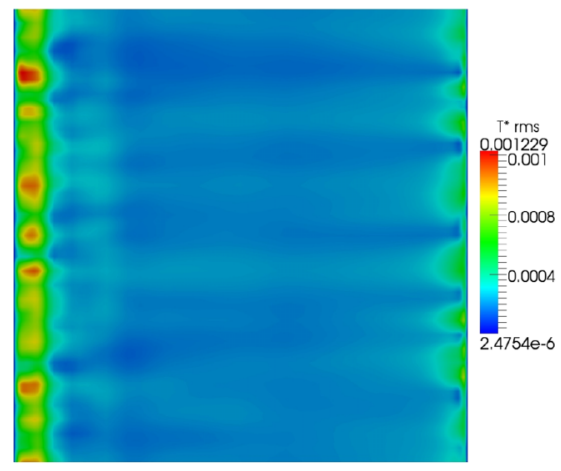

(c)

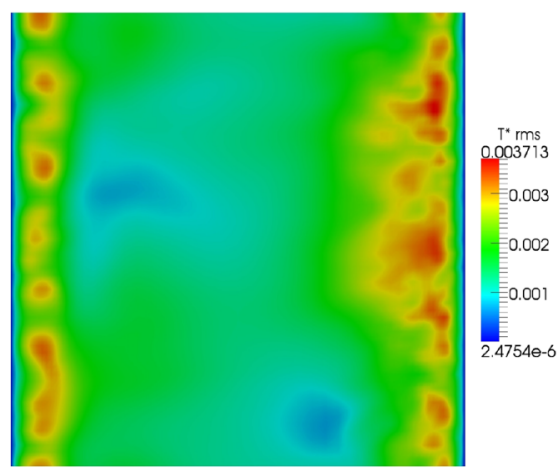

(b)

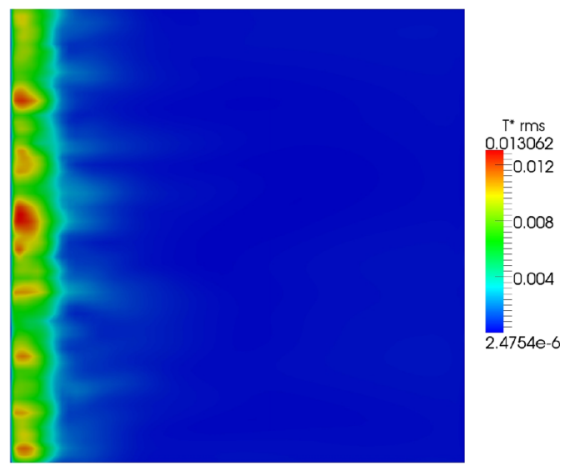

(d)

FIG. 18. Temperature instantaneous fluctuations on the interface between fluid and top wall, $T^{*}=T^{\prime} / \Delta T$. (a) Neosyle. (b) Lead. (c) Concrete stone. (d) Glass wool. 
Looking at Fig. 17(b), we can identify three thermal areas in the top solid medium. Note that the description for the upper part of the cavity repeats in an anti-symmetric way for the lower part:

The impact zone: $(0 \lesssim x / L \lesssim 0.2)$ : the hot fluid plume hits against the cavity ceiling. Heat transfer is dominated by the hot incoming flow.

Thermal perturbations in the case of concrete are less-intense and more localized, while, in case of glass wool, they are more intense and distributed. This occurs because glass wool has a smaller $\rho C_{p}$ that allows for a better thermal fluctuation. Also, lead's case presents a maximum of temperature fluctuations in this region. On the contrary, Neosyle exhibits a smoother and qualitatively different profile. In fact, the recirculation bubble appears farther from the vertical wall, and the hot plume can exchange heat with the solid medium over a wider interfacial region, before fluid separation.

The parallel flow region: $(0.2 \lesssim x / L \lesssim 0.8)$ : here, fluid motion is parallel to the horizontal wall. Conductor materials exhibit larger levels of fluctuations, while insulators have almost no fluctuations. Heat transfer is less influenced by convective motion. Indeed, this is a region of lower turbulence level (see Fig. 16) and the thermal fluxes are almost absent (see Figs. 14 and 15) with respect to other regions.

The fall-down region: $(0.8 \lesssim x / L \lesssim 1)$ : here the flow deviates downward and internal solid temperature decreases. In cases of insulators, see Fig. 17(a), a rapid diminution of internal temperature occurs: in case of glass wool, it decays very close to the right wall and temperature fluctuations are practically absent $\left(T_{r m s} \sim 0\right)$; in case of concrete, a small level of temperature fluctuations is present; lead is colder in this region and the interfacial interaction generates more fluctuations; Neosyle presents the smoother behavior.

Finally, the thermal fluctuations at the solid-fluid interface are analyzed. TAR is the key parameter for studying the perturbations at the boundaries, and it relates the degree of interaction between the two phases, as discussed in Sec. III. For the four materials herein considered, TAR differs by orders of magnitude, as reported in Table III.

Fig. 18 shows temperature instantaneous fluctuations at the top solid surface. As the TAR decreases, the interface exhibits a larger level of fluctuations, except for the glass wool case. In materials characterized by smaller TAR, the fluctuations are more spread around the plate, whereas for large values of TAR, fluctuations remain localized in a region close to hot wall where the vertical flow impact the top solid surface.

However, it has to be pointed out that a direct comparison between the materials cannot be performed. In fact, Equation (27) suggests that the solid-fluid heat flux is inversely proportional to the effusivity, and directly proportional to the temperature difference. Since the solid media are all at different thermal evolution stages, the internal temperatures of the materials are quite different from case to case, as already shown in Fig. 17(b). Thus, the effects of different effusivities are overcome by the different temperature gradients.

\section{CONCLUSIONS}

Turbulent natural convection in a enclosure is studied numerically, via large-eddy simulations. A Lagrangian dynamic sub-grid scale model is adopted to determine the turbulent subgrid-scale viscosity and temperature diffusivity, while the conjugate heat transfer between solid media and fluid flow is taken into account by the method developed by Sosnowski et al.$^{20}$ The implementation of the numerical model is carried out within the OpenFOAM framework.

A paradigmatic case is analyzed: a square cavity, filled with air, having two different heated vertical walls and two conductive horizontal boundaries, separated from the outside by insulator blocks. The Rayleigh number is chosen to be $R a=1.58 \times 10^{9}$ as in the previous literature studies.

First, the code is validated against experimental and numerical data given in Refs. 5-7 and 11, respectively. Flow statistics are in good agreement with those reported in the literature. Specific attention is paid to thermal coupling validation. In our numerical experiment, the conductive sheets and the insulator blocks as in the benchmark experiment are taken into account. The temperature 
distribution is correctly reproduced. However, the numerical results and a theoretical analysis of temperature diffusion time show that in the experiment, diffusion process is still in an evolution stage which can elapse over few days. This highlights the fact that sometimes the adiabatic condition commonly used in numerical simulations, and intended to mimic a perfect insulator, may be representative of an asymptotic behavior difficult to reach in real cases.

The model is successively applied to study, in the same flow configuration as in the literature experiments, the impact of different solid media on the flow features and temperature distribution. Specifically, conjugate heat transfer is considered at the top/bottom horizontal plates, usually modeled as perfect insulators. Four materials are considered: two conductors and two insulators. An additional simulation is carried out considering perfect insulating walls, reproduced by applying the adiabatic condition.

The problem of the characteristic time of temperature diffusion in solid media is addressed. The geometrical dimensions of the horizontal plates lead to two different diffusion times for horizontal and vertical direction, respectively. The asymptotic steady-state thermal configuration is reached after a time comparable with the horizontal characteristic time, that could be huge for materials with low thermal diffusivity. In order to perform numerical simulations representative of physical experiments, the initial conditions for the temperature field in the solid and fluid media have been set close to those of typical experiments.

To study the system, in order to circumvent the problem associated with low diffusivity of some materials, leading to an excessive developing time non-affordable in numerical simulations, a four-step procedure is developed. It consists in the use of a temperature advancement strategy based on the artificial reduction of the $C_{p}$ of the solid medium during the early stage of development of the flow field, and to recover its actual value for obtaining the final flow configuration. The same physical time is reproduced in all cases and different degrees of thermal development were thus reached, depending on the characteristics of the solid media; for the high conductor case (Neosyle) and the perfect insulator case only, the system reached the steady state.

The two cases are, hence, considered as representative of the two categories of conductors and insulators. All cases are characterized by anti-symmetry with respect to the diagonals of the cavity and hence, the discussion has been developed for a portion of the domain, invoking anti-symmetry for the remaining part.

Except for perfect insulator, a recirculation bubble close to horizontal walls near the top-left and bottom-right corners is observed.

Further, the presence of conductors produces stronger turbulence on the top-right corner that increases the buoyancy force in that regions and results in high fluid velocity near the horizontal walls.

The core region is always characterized by weak stable stratification and, for conductors, nearly laminar flow developing along horizontally shaped low-speed recirculating flow. Temperature inside horizontal plates presents profile higher then linear distribution for conductors, while for real insulator cases, it is still evolving. Fluctuations at the solid-fluid interface are less intense than in the insulator cases and diffused over the horizontal sheets.

Perfect insulator can be considered as the asymptotic limit for insulating media, when $k \rightarrow 0$. However, the analysis clearly shows that the flow features may be different from real insulating materials when the asymptotic regime has not been reached.

Finally, in the present paper, it has been mentioned that disagreements in the temperature profile in the core region within the cavity may be due to the effects of thermal radiation here not considered. Radiation models are currently under analysis and will be implemented in the near future in our model.

\section{ACKNOWLEDGMENTS}

This research has been carried out using computational facilities procured through the European Regional Development Fund, Project No. ERDF-080 "A Supercomputing Laboratory for the University of Malta." 


\section{APPENDIX: ADDITIONAL VALIDATION ANALYSIS}

In Sec. V, the validation of the numerical model is carried against the literature numerical and experimental data. In order to corroborate the simulation methodology adopted, additional analysis is reported in this appendix: first, mesh and time step dependency are investigated; then, we estimate the mesh size and time step employed with respect to the Kolmogorov length and time scale to detect the range of subgrid-scale parametrized during the simulation.

Mesh and time step dependency: The reference case in absence of CHT, described in Sec. IV D, is reproduced again with four different settings:

1. the mesh resolution is held to $96 \times 96 \times 64$ cells but the Courant number of the simulation is increased to $C o=1$;

2. the mesh is held as in the previous case but the Courant number of the simulation is decreased to $C o=0.25$;

3. the time step is held at $C o=0.5$, but a finer mesh is used, with a resolution of $128 \times 128 \times 64$ points;

4. the time step is held at $C o=0.5$, but a coarser mesh is used, with a resolution of $64 \times 64 \times 64$ points.

The Courant number is defined, cell by cell, as $C o=\Delta t|\bar{u}| / \delta x$, where $\Delta t$ is the time step, $|\bar{u}|$ is the velocity magnitude through the cell, and $\delta x$ is the cell size in the direction of the velocity.

An analysis of grid dependency in the spanwise direction was done by Peng and Davidson ${ }^{11}$ for the same case, hence it is not repeated here. We use the same number of grid points as in Ref. 11 along the spanwise direction in all cases. The results are compared with those of the reference simulation reported in Sec. V, which has a grid resolution of $96 \times 96 \times 64$ points and Co $=0.5$.

The simulations with finer and coarser meshes are stretched in such way that the first cell near the wall has the same width as in the reference simulation. After a statistical steady state is reached, the statistics are computed during a period of time roughly corresponding to one turnover time of the main cavity vortex, for all simulations.

Fig. 19(a) shows the non-dimensional first and second order statistics for the velocity field. The quantities are plotted near the hot wall, along the horizontal center-line $(y / L=0.5)$ within the cavity. The results obtained with different time steps practically collapse over the same curves; thus, the results are substantially time step independent for a wide range of values of the Courant number.

As regards space resolution, simulation with finer mesh slightly differs from the reference case: a slightly higher peak of the vertical velocity is observed together with second-order statistics closer to the experimental data. On the contrary, the simulation with the coarser mesh exhibits less accurate results. The vertical velocity profile has slightly higher peak and lower values farther from the wall. The RMS of the vertical velocity is overestimated while that of the horizontal one is underestimated.

Fig. 19(b) reports the mean and RMS temperature profile along the same horizontal center-line as in Fig. 19(a). The temperature profiles do not vary from case to case, while the second order statistics of temperature obtained with the coarse mesh case are lower than in the other cases.

Overall, the grid size used in our study, which is the same as that used in Ref. 11, is able to reproduce the first and second-order statistics of the flow accurately. The choice of the time step within a wide range of values of Courant numbers does not affect the quality of the results.

Turbulent length scales: To get information on the range of spatial scales not resolved in the simulation, the Kolmogorov turbulent length-scale $\eta=\left(v^{3} /\langle\epsilon\rangle\right)^{1 / 4}$, where $\epsilon$ is the turbulent dissipation rate, is computed for the reference simulation. The dissipation rate $\epsilon$ was calculated considering the sum of resolved and SGS contributions.

Fig. 20 shows a contour plot of the ratio $\bar{\Delta} / \eta$ between the local cell width and the turbulent length scale $\eta$. The figure clearly shows that turbulence is present near the walls. The values of $\bar{\Delta} / \eta \sim 1$ in the core region of the cavity must be intended as representative of low-level turbulence, nearly laminar, region. The local cell width is one order of magnitude larger than $\eta$ in the boundary 

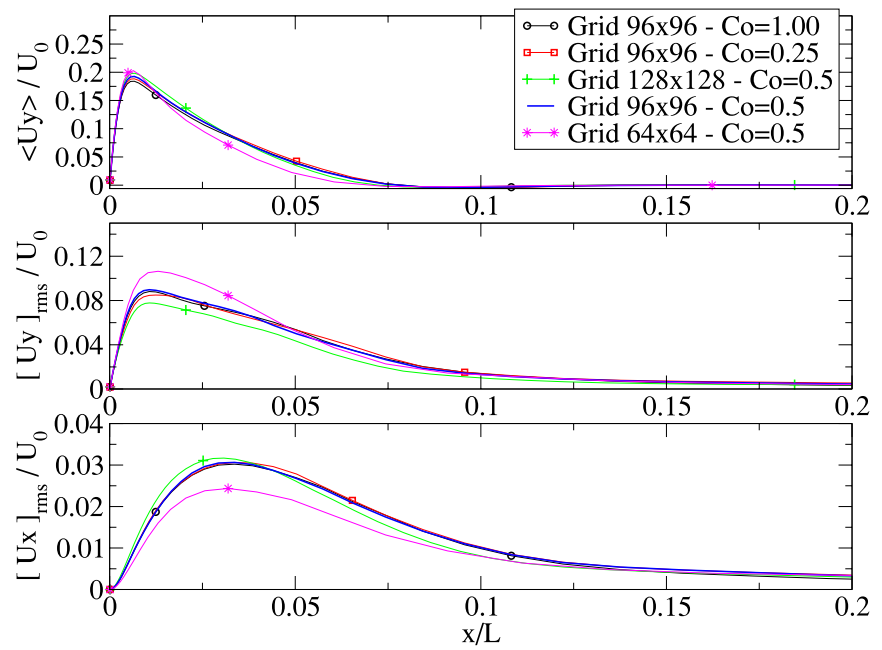

(a)
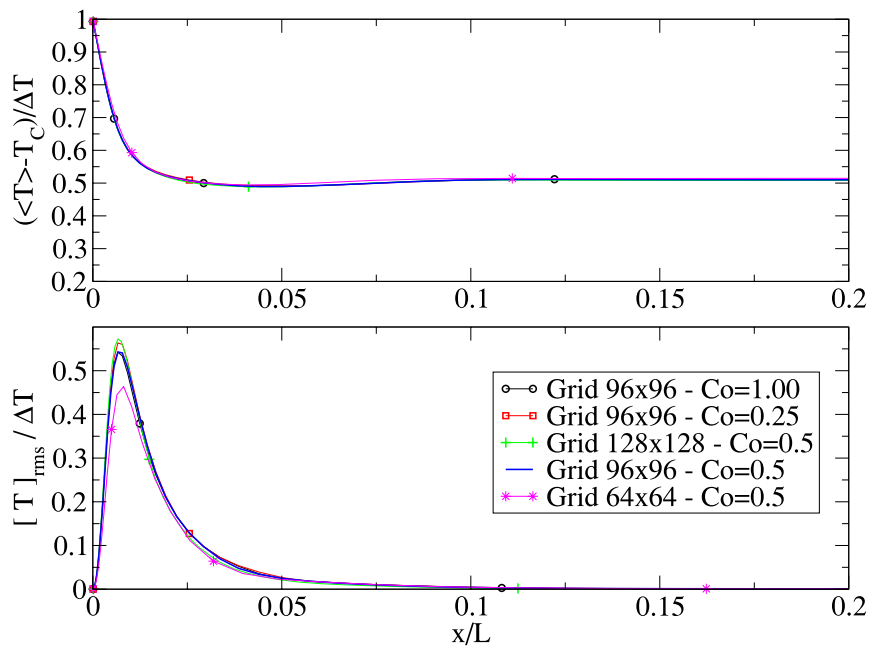

(b)

FIG. 19. Comparison of non-dimensional quantities for different mesh resolutions and time steps. Quantities are plotted along an horizontal line at height $y / L=0.5$, near the hot wall. (a) Fluid flow quantities: (top) vertical velocity profile; (center) vertical RMS; (bottom) horizontal RMS. (b) Temperature profile and root-mean square: (top) temperature profile; (bottom) temperature root-mean square.

layers developing near the vertical walls, whereas the ratio decreases close to the horizontal walls, where the boundary layer is less energetic.

Fig. 21 reports the time power spectra of the resolved turbulent kinetic energy, computed by using the Fourier transform of data recorded at three probes located near the vertical hot wall at $x / L=0.007$ (approximately where the vertical velocity has a peak), at $z / L=0.5$ and at three different heights $y / L=0.2,0.5,0.8$. A well-defined inertial subrange $E(\omega) \sim \omega^{-5 / 3}$ cannot be detected, indicating that this is a low Reynolds number turbulent flow where the production range of scales is not completely separated from the dissipation one. This is typical of wall-resolving LES where the near wall thin boundary layer has to be appropriately resolved.

The ratio between the simulation time step $\Delta t$ and the Kolmogorov turbulent time scale $\tau_{k}=\sqrt{v / \epsilon}$ at the three locations is $\Delta t / \tau_{k}<1$, indicating that the whole turbulent spectrum is resolved in time. This is typical of LES of low Reynolds number flows run with values of Courant number smaller than unity. 


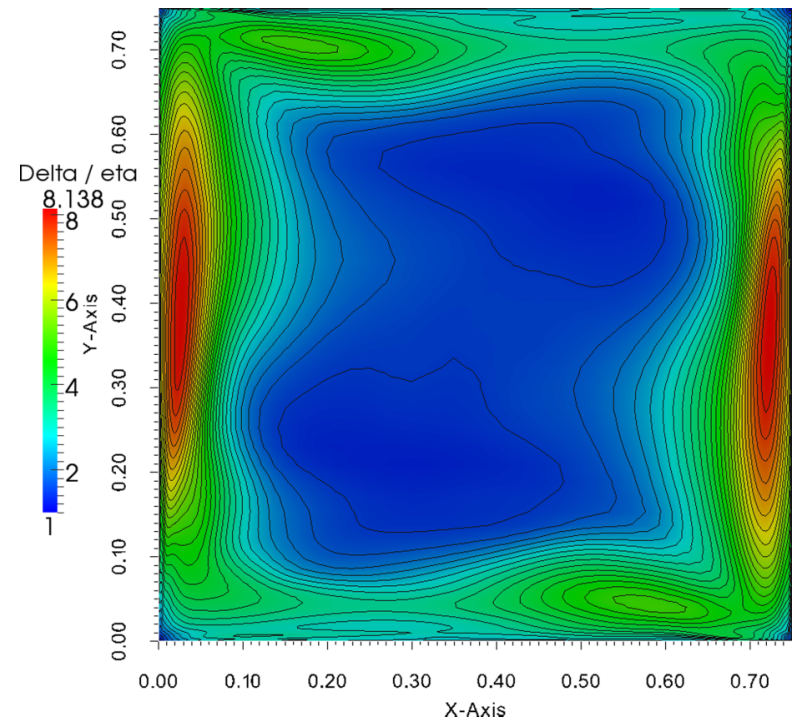

FIG. 20. Contour plot of the ratio $\bar{\Delta} / \eta$ between the local cell width and the Kolmogorov length scale.

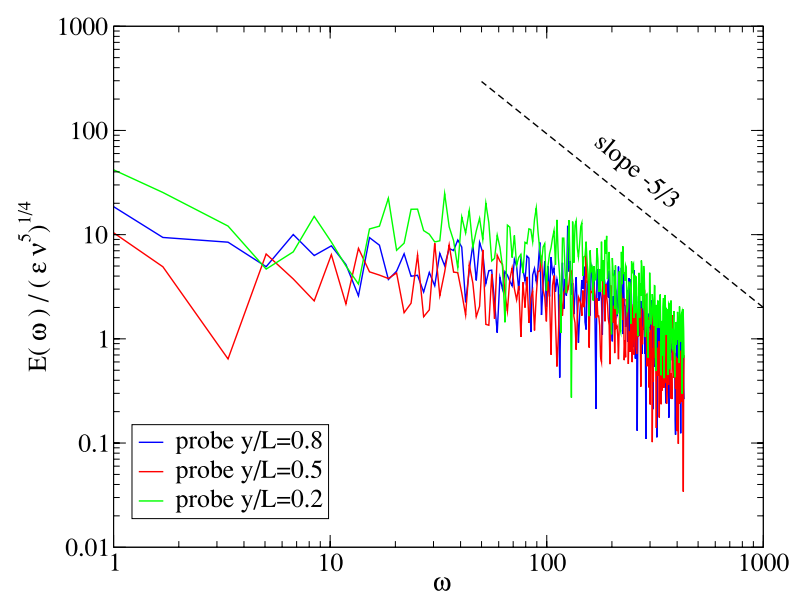

FIG. 21. Normalized time power spectra of the resolved turbulent kinetic energy $\kappa=\left\langle u_{i}^{\prime} u_{i}^{\prime}\right\rangle / 2$. The data used are recorded at three probes located near the hot vertical wall $(x / L=0.007)$, in the mid-plain $(z / L=0.5)$, at three characteristics heights $(y / L=0.2,0.5,0.8)$.

To summarize, the analysis suggests that most turbulent scales are directly resolved in the simulation, which can be classified as quasi-dns according to the definition given in Spalart et al. ${ }^{32}$

${ }^{1}$ Among the others, we can refer to Marín ${ }^{28}$ for a brief derivation of this formula.

${ }^{2}$ Fancy name compound of ancient Greek words véos (neos) "new," and $v \dot{\lambda} \eta$ (yle) "material, substance."

${ }^{3} \mathrm{~S}$. Mergui and F. Penot, "Convection naturelle en cavité carrée différentiellement chauffée: Investigation expérimentale à $R a=1.69 \times 10^{9}$, , Int. J. Heat Mass Transfer 39, 563 (1996).

${ }^{4}$ J. Salat, S. Xin, P. Joubert, A. Sergent, F. Penot, and P. Le Qur, "Experimental and numerical investigation of turbulent natural convection in a large air-filled cavity," Int. J. Heat Fluid Flow 25, 824 (2004).

${ }^{5}$ Y. S. Tian and T. G. Karayiannis, "Low turbulence natural convection in an air filled square cavity. Part I: The thermal and fluid flow fields," Int. J. Heat Mass Transfer 43, 849 (2000).

${ }^{6}$ Y. S. Tian and T. G. Karayiannis, "Low turbulence natural convection in an air filled square cavity. Part II: The turbulence quantities," Int. J. Heat Mass Transfer 43, 867 (2000).

${ }^{7}$ F. Ampofo and T. G. Karayiannis, "Experimental benchmark data for turbulent natural convection in air filled square cavity," Int. J. Heat Mass Transfer 46, 3551 (2003).

${ }^{8}$ M. Omri and N. Galanis, "Numerical analysis of turbulent buoyant flows in enclosures: Influence of grid and boundary conditions," Int. J. Therm. Sci. 46, 727 (2007).

${ }^{9}$ K. J. Hsieh and F. S. Lien, "Numerical modeling of buoyancy-driven turbulent flows in enclosures," Int. J. Heat Fluid Flow 25, 659 (2004). 
${ }^{10}$ A. Ibrahim, D. Saury, and D. Lemonnier, "Coupling of turbulent natural convection with radiation in an air-filled differentially-heated cavity at $R a=1.5 \times 10^{9}$," Comput. Fluids 88, 115 (2013).

${ }^{11}$ S. H. Peng and L. Davidson, "Large eddy simulation for turbulent buoyant flow in a confined cavity," Int. J. Heat Fluid Flow 22, 323 (2001).

${ }^{12}$ C. Bosshard, A. Dehbi, M. Deville, E. Leriche, R. Puragliesi, and A. Soldati, "Large eddy simulation of the differentially heated cubic cavity flow by the spectral element method," Comput. Fluids 86, 210 (2013).

${ }^{13}$ C. Zimmermann and R. Groll, "Modelling turbulent heat transfer in a natural convection flow," J. Appl. Math. Phys. 2, 662 (2014).

${ }^{14}$ A. Dorfman and Z. Renner, "Conjugate problems in convective heat transfer: Review," Math. Probl. Eng. 2009, 1.

${ }^{15}$ F. Duchaine, A. Corpron, L. Pons, V. Moureau, F. Nicoud, and T. Poinsot, "Development and assessment of a coupled strategy for conjugate heat transfer with Large Eddy Simulation: Application to a cooled turbine blade," Int. J. Heat Fluid Flow 30, 1129 (2009).

${ }^{16}$ F. Duchaine, S. Mendez, F. Nicoud, A. Corpron, V. Moureau, and T. Poinsot, "Conjugate heat transfer with Large Eddy Simulation for gas turbine components," C. R. Mec. 337, 550 (2009).

${ }^{17}$ I. Tiselj, R. Bergant, B. Mavko, I. Bajsić, and G. Hetsroni, "DNS of turbulent heat transfer in channel flow with heat conduction in the solid wall," J. Heat Transfer 123, 849 (2001).

${ }^{18}$ A. Garai, J. Kleissl, and S. Sarkar, "Flow and heat transfer in convectively unstable turbulent channel flow with solid-wall heat conduction," J. Fluid Mech. 757, 57 (2014).

${ }^{19}$ A. Quarteroni and A. Valli, Domain Decomposition Methods for Partial Differential Equations (Oxford University Press, 1999).

${ }^{20}$ P. Sosnowski, A. Petronio, and V. Armenio, "Numerical model for thin liquid film with evaporation and condensation on solid surfaces in a systems with conjugated heat transfer," Int. J. Heat Mass Transfer 66, 382 (2013).

${ }^{21}$ J. Smagorinsky, "General circulation experiments with the primitive equations: I. The basic experiment," Mon. Weather Rev. 91, 99 (1963).

${ }^{22}$ M. Germano, U. Piomelli, P. Moin, and W. Cabot, "A dynamic subgrid-scale eddy viscosity model," Phys. Fluids A 3 , 1760-1765 (1991).

${ }^{23}$ D. Lilly, "A proposed modification of Germano subgrid-scale closure method," Phys. Fluids A 4, 633-635 (1992).

${ }^{24}$ C. Meneveau, T. S. Lund, and W. H. Cabot, "A Lagrangian dynamic subgrid-scale model of turbulence," J. Fluid Mech. 319, 353-385 (1996).

${ }^{25}$ V. Armenio and S. Sarkar, "An investigation of stably stratified turbulent channel flow using large-eddy simulation," J. Fluid Mech. 459, 1-42 (2002).

${ }^{26}$ R. I. Issa, "Solution of the implicitly discretized fluid flow equations by operator-splitting," J. Comput. Phys. 62, 40-65 (1985).

${ }^{27}$ R. I. Issa, A. D. Gosman, and A. P. Watkins, "The computation of compressible and incompressible recirculating flows by a non-iterative implicit scheme," J. Comput. Phys. 62, 66-82 (1986).

${ }^{28}$ E. Marín, "Characteristic dimension for heat transfer," Lat. Am. J. Phys. Educ. 4(1), 56-60 (2010).

${ }^{29}$ P. Moin and K. Mahesh, "Direct numerical simulation: A tool in turbulence research," Annu. Rev. Fluid Mech. 30, 539-578 (1998).

${ }^{30}$ American Society of Heating and Refrigerating and Air-Conditioning Engineers, ASHRAE Handbook Fundamentals, SI ed. (Ashrae, Atlanta, 2005).

${ }^{31}$ S. B. Pope, Turbulent Flows (Cambridge University Press, 2000).

${ }^{32}$ P. R. Spalart, W.-H. Jou, M. Strelets, and S. R. Allmaras, "Comments on the feasibility of LES for wings, and on a hybrid RANS/LES approach," in First AFOSR International Conference on DNS/LES, Ruston, LA, 4-8 August, Advances in DNS/LES edited by C. Liu and Z. Liu (Greyden Press, Columbus, OH, 1997). 